Generalized false positive models

\title{
A generalized observation confirmation model to account for false positive error in species
}

\section{detection-nondetection data}

John D. J. Clare ${ }^{1 *}$, Benjamin Zuckerberg ${ }^{1}$, and Philip A. Townsend ${ }^{1}$

${ }^{1}$ Department of Forest and Wildlife Ecology, University of Wisconsin - Madison, Madison,

Wisconsin

*jclare2@ wisc.edu; 1630 Linden Drive, Madison, Wisconsin 53706

Running head: Generalized false positive models 
Generalized false positive models

\section{Abstract}

Spatially-indexed repeated detection-nondetection data is widely collected by ecologists interested in estimating parameters associated with species distribution, relative abundance, phenology, and more while accounting for imperfect detection. Recent model development has focused on accounting for false positive error as well, given growing recognition that misclassification is common across many sampling protocols. To date, however, the development of model-based solutions to false positive error has been largely restricted to occupancy models. We describe a general form of the observation confirmation protocol originally described for occupancy estimation that permits investigators to flexibly and intuitively extend several models for detection-nondetection data to account for false positive error. Simulation results demonstrate that estimators for relative abundance and arrival time exhibit relative bias greater than $20 \%$ under realistic levels of false positive prevalence (e.g., $5 \%$ of detections are false positive). Bias increases as true and false positives occur in more distinct places or times, but can also be sensitive to the values of the state variables of interest, sampling design, and sampling efficiency. Results from an empirical study focusing on patterns of gray fox relative abundance across Wisconsin, USA suggest that false positive error can also distort estimated spatial patterns often used to guide decision-making. The extended estimators described within typically improve performance at any level of confirmation, and when false positive error occurs at random and constitutes less than $10 \%$ of all detections, the estimators are essentially unbiased when more than 50 observations can be confirmed as true or false positives. The generalized form of the observation-confirmation protocol is a flexible model-based solution to false positive error useful for researchers collecting data with sampling devices like trail or 
Generalized false positive models

23 smartphone cameras, acoustic recorders, or other techniques where classifications can be

24 reviewed post-hoc.

\section{Introduction}

Detection non-detection data is widely collected in ecology. The importance of collecting repeated individually-indexed detection-nondetection data to account for observation error associated with imperfect detection has been recognized for decades (e.g., Otis et al. 1978). More recently, spatially-structured models that use repeated observations of species occurrence at specific locations to account for imperfect detection have seen rapid development and increasing use. These models have greatly expanded what can estimated with detection-nondetection data, permitting inference about several ecological state variables such as species distribution, abundance, phenology, density, and associated dynamics (MacKenzie et al. 2002, Royle and Nichols 2003, Roth et al. 2014, Ramsey et al. 2015, Rossman et al. 2016).

However, a growing body of evidence suggests that imperfect detection is not the only important source of observation error in detection-nondetection data. Across a variety of species classification or identification protocols, false positive error (e.g., a species is falsely observed at a time or place when not present) has been shown to interspecifically vary from nearly negligible to constituting $20 \%$ of observations or more (Simons et al. 2007, McClintock et al. 2010a, Swanson et al. 2016, Norouzzadeh et al. 2018). Simulation results have shown that even relatively small amounts of false positive error can severely bias species distribution estimators

42 that assume it does not occur (e.g., absolute error in the proportion of occupied sites $>0.1$, Miller

43 et al. 2011, Ruiz-Gutiérrez et al. 2016). This has motivated investigators to adopt a variety of

44 strategies aimed at ameliorating biases induced by false positive error. The most thorough 45 strategy is to implement a complete data review after collection (e.g., Gardiner et al. 2012), but 
Generalized false positive models

this can become burdensome with the sizable datasets produced by automated detection devices or citizen scientists (Ruiz-Gutiérrez et al. 2016). In the interest of efficiency, investigators often turn to several other approaches such as using partial reviews to develop indicators or algorithms to identify misclassified data, simplifying classification tasks, or providing additional training (data) to human (or computer-based) classifiers (Swanson et al. 2016, see review by Kosmala et al. 2016). Although these approaches can greatly improve data quality, they suffer certain limitations. It can be time-consuming to quantify improvements in data quality resulting from protocol or training changes and to determine how accurate raw data or error indicators need to be to achieve desired inferential reliability (Clare et al. 2019). Furthermore, appropriately propagating indicator uncertainty or any remaining data error into subsequent estimate uncertainty is not always straightforward. Model-based solutions may be the most elegant way to ameliorate false positive error, Mackenzie et al. (2002) conceptualizes observed absences as a mixture of true and false negatives, and subsequent extensions all conceptualize observed occurrences as also arising from

63 a mixture of true and false positives. The varied protocols primarily differ with regard to how the

64 probability of a false positive observation is informed within the estimation process. Under the

65 'full' estimator described by Royle and Link (2006), all observed occurrences are of unknown reliability, and disentangling the false negative and false positive mixtures requires multiple

67 constraints. Subsequent developments leverage auxiliary data to directly inform parts of the positive and/or negative mixtures, like additional error-free observations provided by an 
Generalized false positive models

additional sampling method (site-confirmation protocol), experimental trials for classification performance under controlled settings (calibration protocol) or confirming a subset of the observations post-hoc (observation confirmation protocol; Miller et al. 2011, Chambert et al. 2015, Ferguson et al. 2015, Ruiz-Gutiérrez et al. 2016). An advantage of these model-based solutions is that they reduce positive estimator bias associated with false positives while also propagating uncertainty in observation reliability into estimator uncertainty.

The rapid development of model extensions to accommodate false positive error across a variety of sampling situations suggests that many researchers recognize that false positives are common and problematic. It is surprising and concerning, then, that model development and application has largely been restricted to occupancy estimation. False positives presumably induce bias in any parameter or state variable estimated with detection-nondetection data, and the cost of biased estimation is arguable greater for many parameters or state variables other than distribution (e.g. species abundance).

We suspect that a major barrier to broader adoption and development is that many of the specific conditional probability statements described within false positive occupancy models are not intuitive for many ecologists to translate into other models using similar data. Here, we clarify the flexibility of model-based solutions to false positive error within detectionnondetection data using basic laws of probability. We focus on the observation-confirmation protocol, which is most pragmatic formulation for investigators reliant upon sampling techniques that produce data that can be reviewed a posteriori (images, audio recordings, physical specimens like scats, etc.) and is arguably the most efficient model-based solution (Chambert et al. 2015). Here, we present a generalizable model structure, demonstrate that standard and false positive occupancy models are special cases of said structure, and present extensions of models 
Generalized false positive models

92 for relative abundance or phenological parameters like migratory arrival or emergence from

93 hibernation that account for false positive error. We then use simulation to demonstrate that false

94 positive error compromises estimation of relative abundance and migratory arrival, and that our

95 model extensions commonly improve inference.

\section{The Generalized Model Form}

97 Throughout, we assume a data matrix of binary observations $\boldsymbol{y}$ that corresponds to the detection

98 or nondetection of some species (or other phenomena of interest) at $i=1,2, \ldots \mathrm{R}$ locations over $j$

$99=1,2 \ldots$ T discrete sampling intervals. We also assume $y_{i, j} \sim$ Bernoulli $(\theta)$, where $y_{i, j}=1$ if a

100 species is observed and 0 otherwise; $\theta$ describes the probability of a positive observation.

101 Commonly, $\theta$ is likely to be a mixture of the respective probabilities of true positive observations

102 that are the research focus $\left(\theta_{t p}\right)$ and false positive observations $\left(\theta_{f p}\right)$; decomposing these

103 components allows one to make exclusive inference about $\theta_{t p}$. Assuming that true and false

104 positive observations are independent, the specification of the mixture of true and false positives

105 can be derived from addition rules for probabilities. True and false positives might be mutually

106 exclusive events such that $y_{i, j} \sim$ Bernoulli $\left(\theta_{t p}+\theta_{f p}\right)$, implying that a positive observation is either

107 a true positive or a true positive. Alternatively, a specific binary outcome might include only true

108 positive observations, only false positive observations, or both, in which case $y_{i, j} \sim$ Bernoulli

$109\left(\theta_{t p} \times\left[1-\theta_{f p}\right]+\theta_{f p} \times\left[1-\theta_{t p}\right]+\theta_{t p} \times \theta_{f p}\right)$. After factorization, this simplifies to $y_{i, j} \sim \operatorname{Bernoulli}\left(\theta_{t p}+\right.$

$\left.110 \theta_{f p}-\left[\theta_{t p} \times \theta_{f p}\right]\right)$. These probabilistic statements constitute a generalized structure for modeling

111 true and false positive probabilities in binary data.

Many models commonly used by ecologists can be described using the generalized

113 structure. A generalized (logit) linear model for species occurrence could be described as a case

114 that assumes logit $\left(\theta_{t p, i}\right)=\boldsymbol{X}_{\boldsymbol{i}}^{\boldsymbol{T}} \boldsymbol{\beta}$ and $\theta_{f p}=0$. A generalized (logit) linear mixed model for repeated 
Generalized false positive models

115 species occurrences is a case assuming logit $\left(\theta_{t p, i}\right)=\boldsymbol{X}_{\boldsymbol{i}}^{\boldsymbol{T}} \boldsymbol{\beta}_{\boldsymbol{i}}, \theta_{f p}=0$, and $\boldsymbol{\beta}_{\boldsymbol{i}} \sim \operatorname{Normal}\left(\boldsymbol{\mu}_{\boldsymbol{\beta}}, \boldsymbol{\sigma}_{\boldsymbol{\beta}}\right)$. The occupancy model described by MacKenzie et al. (2002) can be described as a case assuming $\theta_{f p}$

$117=0$ and $\theta_{t p, i}=z_{i} \times p$, where $z_{i}$ is the latent occupancy state of site $i, z_{i} \sim \operatorname{Bernoulli}(\psi), p$ is the

118 probability of detecting an organism at a site given that it is present, and both $p$ and $\psi$ might be incorrectly. Rather, the estimands associated with $\theta_{t p, i}$ actually describe a mixture of true and false positive probabilities, either $\theta_{t p, i}+\theta_{f p}$, or $\theta_{t p}+\theta_{f p}-\left(\theta_{t p} \times \theta_{f p}\right)$. confirmation, and calibration models follow the mutually exclusive specification, where $y_{i, j} \sim$ models, all three generally treat $\theta_{f p, i}=\left(1-z_{i}\right) \times p_{10}$ and $\theta_{t p, i}=z_{i} \times p_{11}$, where $p_{11}$ is the probability fact, because $\theta_{f p, i}$ and $\theta_{t p, i}$ are dependent upon the state $z_{i}$, true and false positive detections are mutually exclusive not only within specific cells $y_{i, j}$, but across sites (rows) $\boldsymbol{y}_{i, . .}$ The observationconfirmation model does not require that true and false positives be mutually exclusive, and we focus on extending this model below.

\section{The Observation Confirmation Protocol}

134 As originally described by Chambert et al. (2015), the observation confirmation model addresses an estimation problem where the purpose is to estimate the binary occurrence state within a finite sample of sites $\left(z_{i}\right)$ or the population level probability of occurrence $\psi$, assuming $z_{i} \sim$ Bernoulli $(\psi)$. Within some subset of sampling intervals $j$ at specific sites, some observations $\left(v_{i, j}\right)$ have 
Generalized false positive models

138 been verified as either containing only true positives $\left(v_{i, j}=0\right)$, only false positives $\left(v_{i, j}=1\right)$, both $139\left(v_{i, j}=2\right)$, or no positive observations $\left(v_{i, j}=3\right)$; note that we order the terms differently than

140 Chambert et al. (2015). These verified observations might include physical samples (hair or

141 scat), images, audio or video recordings, or any other data type that can subsequently be

142 confirmed via expert evaluation, laboratory analysis, or other means. The confirmed samples $v_{i, j}$

$143 \sim$ Categorical $(\boldsymbol{\Omega})$, where the elements of $\boldsymbol{\Omega}$ are conditional on whether the species occurs at site

$144 i$ or not. If $z_{i}=1$ (species occurs), then $\boldsymbol{\Omega}_{\mathbf{i}}=\left[\left\{\left(s_{1} \times\left(1-s_{0}\right)\right\}\left\{s_{0} \times\left(1-s_{1}\right)\right\}\left\{s_{1} \times s_{0}\right\}\left\{\left(1-s_{1}\right) \times(1\right.\right.\right.$

$\left.\left.\left.145-s_{0}\right)\right\}\right]$. If $z_{i}=0$, the only possible outcomes are a false positive detection or no detection, and $\boldsymbol{\Omega}_{\mathbf{i}}$

$146=\left[\{0\}\left\{s_{0}\right\}\{0\}\left\{1-s_{0}\right\}\right]$. Here $s_{1}$ and $s_{0}$ represent the respective probabilities that a sampling

147 interval contains $>0$ true positive or false positive observations conditional upon the occurrence

148 state. The similar conditional statement for unconfirmed data is $y_{i, j} \sim$ Bernoulli $\left(z_{i} \times p_{11}+\left(1-z_{i}\right)\right.$

$\left.149 \times p_{10}\right)$. If present, a species can either be truly or falsely detected $\left(y_{i, j}=1 \mid z_{i}=1\right)$ with probability

$150 p_{11}=s_{1}+s_{0}-\left(s_{1} \times s_{0}\right)$, and if not $\left(y_{i, j}=1 \mid z_{i}=0\right)$, it can only be falsely detected with probability

$151 p_{10}=s_{0}$.

The observation confirmation occupancy model thus follows a non-exclusive form of the

153 general model based upon addition rules for probabilities. The first three elements of $\boldsymbol{\Omega}$ given

that $z_{i}=1$ in mirror the constituent terms in the general model $\theta_{t p}\left(1-\theta_{f p}\right)+\theta_{f p}\left(1-\theta_{t p}\right)+\theta_{f p} \theta_{t p}$, and

the statement for $p_{11}$ mirrors the factorized $\theta_{t p}+\theta_{f p}-\left(\theta_{t p} \theta_{f p}\right)$. In fact, the observation

156 confirmation model can be described as a specific case of the generalized structure in which $\theta_{t p, i}$

$157=z_{i} \times s_{1}$ and $\theta_{f p}=\mathrm{s}_{0}$. The row vector $\boldsymbol{\Omega}_{\mathbf{i}}$ is then specified as $=\left[\left\{\theta_{t p, i} \times\left(1-\theta_{f p}\right)\right\}\left\{\theta_{f p} \times\left(1-\theta_{t p, i}\right)\right\}\{\right.$

$\left.\left.158 \theta_{f p} \times \theta_{t p, i}\right\}\left\{\left(1-\theta_{t p, i}\right) \times\left(1-\theta_{f p}\right)\right\}\right]$. As before, $v_{i, j} \sim$ Categorical $\left(\mathbf{\Omega}_{\mathbf{i}}\right)$. In turn, $y_{i, j} \sim$ Bernoulli $(1-$

$\left.159 \Omega_{4, i}\right)$; which is exactly equivalent to describing $y_{i, j} \sim$ Bernoulli $\left(\theta_{t p, i}+\theta_{f p}-\left[\theta_{t p, i} \times \theta_{f p}\right]\right)$. In full, a

160 hierarchical description of the complete data likelihood is: 
Generalized false positive models

161

162

163

164

165

166

167

168

169

170

171

172

173

174

175

176

177

178

179

180

$$
z_{i} \sim \text { Bernoulli }(\psi)
$$

$$
\theta_{t p, i}=z_{i} \times s_{1}
$$

$$
\theta_{f p}=s_{0}
$$

$$
\boldsymbol{\Omega}_{\boldsymbol{i}}=\left[\left\{\theta_{t p, i} \times\left(1-\theta_{f p}\right)\right\}\left\{\theta_{f p} \times\left(1-\theta_{t p, i}\right)\right\}\left\{\theta_{f p} \times \theta_{t p, i}\right\}\left\{\left(1-\theta_{t p, i}\right) \times\left(1-\theta_{f p}\right)\right\}\right]
$$

$v_{i, j} \sim$ Categorical $\left(\boldsymbol{\Omega}_{i}\right)$

$y_{i, j} \sim \operatorname{Bernoulli}\left(1-\Omega_{4, i}\right)$

\section{Extending the Observation Confirmation Protocol to other Models}

One outcome of defining the observation confirmation protocol using the general model structure is that it clarifies extension to other models reliant upon detection-nondetection data.

One simply needs to update the definition of $\boldsymbol{\theta}_{t}$ to reflect the full probabilistic statement for a positive outcome using whatever particular model the investigator is interested in fitting (Figure 1). We describe two example extensions in the main text.

Royle-Nichols Model

Royle and Nichols (2003, RN hereafter) describe the unconditional probability of detection (i.e., $\left.\theta_{t p, i}\right)$ as $1-(1-r)^{N_{i}}$, where $r$ is the probability of detecting an individual during a sampling interval and $N_{i} \sim$ Poisson $(\lambda)$ denotes the abundance of a species at site $i$. The hierarchical likelihood for a version incorporating false positives following the observationconfirmation protocol is then:

$$
\theta_{t p, i}=1-(1-r)^{N_{i}}
$$


Generalized false positive models

181

182

183

185

186

187

188

189

190

191

192

193

194

195

196

197

198

$$
\theta_{f p}=\mathrm{s}_{0}
$$

$$
\boldsymbol{\Omega}_{i}=\left[\left\{\theta_{t p, i} \times\left(1-\theta_{f p}\right)\right\}\left\{\theta_{f p} \times\left(1-\theta_{t p, i}\right)\right\}\left\{\theta_{f p} \times \theta_{t p, i}\right\}\left\{\left(1-\theta_{t p, i}\right) \times\left(1-\theta_{f p}\right)\right\}\right]
$$

$v_{i, j} \sim$ Categorical $\left(\boldsymbol{\Omega}_{i}\right)$

$$
y_{i, j} \sim \operatorname{Bernoulli}\left(1-\Omega_{4, i}\right)
$$

Phenological 'arrival' model

Incorporating false positives following the observation-confirmation protocol within an occupancy model designed to estimate the timing of some ephemeral phenomena such as migration arrival or emergence from torpor (Roth et al. 2014, arrival model hereafter) follows Chambert et al.'s (2015) description except that organisms can only be truly detected during sampling intervals at occupied sites after arrival. Thus, $\boldsymbol{\theta}_{t p}$ must be described using indexing for $i$ locations and $j$ time periods (i.e., $\theta_{t p, i, j}$ ). Let arrival time at site $i$ be denoted as $x_{i}$ and assume that $x_{i} \sim$ Poisson $(\varphi)$. To simplify presentation, we define $x_{i}$ in terms of sampling intervals $j$ rather than specific dates. The hierarchical likelihood is:

$$
\boldsymbol{\Omega}_{\mathbf{i}, \mathbf{j}}=\left[\left\{\theta_{t p, i, j}\left(1-\theta_{f p}\right)\right\}\left\{\theta_{f p}\left(1-\theta_{t p, i, j}\right)\right\}\left\{\theta_{f p} \theta_{t p, i, j}\right\}\left\{\left(1-\theta_{t p, i, j}\right) \times\left(1-\theta_{f p}\right)\right\}\right]
$$


Generalized false positive models

199 Here, $\mathrm{I}\left(j \geq x_{i}\right)$ is an indicator function denoting whether the species has arrived at site $i$. As

200 before, confirmed and unconfirmed observations can be described as $v_{i, j} \sim \operatorname{Categorical}\left(\boldsymbol{\Omega}_{\mathbf{i}, \mathbf{j}}\right)$ and

$201 y_{i, j} \sim \operatorname{Bernoulli}\left(1-\Omega_{4, \mathrm{i}, \mathrm{j}}\right)$.

Only a small sample of the possible extensions are described above. Any number of dynamic or integrated models that incorporate repeated detection-nondetection data can be extended to accommodate false positive error following the observation-confirmation protocol simply by defining $\boldsymbol{\theta}_{t}$ using the model described in the initial paper.

In principle, it is also possible to describe false positive extensions to the $\mathrm{RN}$ or arrival models following the site-confirmation or calibration protocol and the full estimator. The sitefalse positives are only possible at sites where $N_{i}=0$, and for the arrival model, false positives

210 are only possible at occupied sites during time periods prior to arrival or at unoccupied sites. The 211 general way to extend these models is to define $\boldsymbol{\theta}_{\boldsymbol{t}}$ following the initial description of the model

212 of interest, and to condition $\theta_{f p}=0$ when $\theta_{t p, \mathrm{i}}>0$ and $>0$ when $\theta_{t p, \mathrm{i}}=0$ (see Appendix S1).

213 However, these protocols depend upon $\theta_{t p, \mathrm{i}}=0$ having functional support. An example where $\theta_{t p, \mathrm{i}}$

$214=0$ is impossible and where extension is limited to the observation-confirmation protocol is the

215 spatially explicit variant of Royle and Nichols' (2003) model (Ramsey et al. 2015; see

216 description in Appendix S1).

217 Exploring Base Model Sensitivity to Error and Performance of Extended Models

218 We undertook a proof of concept simulation study to evaluate the baseline sensitivity of the RN

219 and arrival models to different amounts of false positive error and the performance of the

220 described extensions. Throughout, we considered a fixed level of effort-200 spatial replicates 
Generalized false positive models

221 with 20 temporal replicates each. Given the wide range of potential variability in parameter

222 values, functional forms, and sensitivity to observation aggregation (Balantic and Donovan

223 2019), this is not an exhaustive assessment of estimator properties.

225 We first considered 6 different simulation scenarios, within which we generated 300 replicate 226 datasets with site-specific abundances $\mathrm{N}_{i, \text { sim }} \sim$ Poisson $\left(\lambda_{i, \text { sim }}\right)$ and $\log \left(\lambda_{i, \text { sim }}\right)=\beta_{0}+\beta_{1} X_{1, i, s i m}$,

227 where $X_{1, i, s i m} \sim \mathrm{N}(0,1), \beta_{0}=0$ or -1.5 (3 scenarios each), and $\beta_{1}=1$; sim indexes a particular 228 simulation replicate. Thus, at a site with an average simulated covariate $\left(X_{1, \mathrm{i}}=0\right)$, expected 229 abundance was respectively 1 animal or roughly 0.25 animals. These values were chosen 230 because the RN model tends to perform best when site-specific abundance is low (Kéry and 231 Royle 2016, p. 302) and is perhaps most commonly applied for low-density species. We first 232 generated 'true' detection data as Bernoulli $\left(p_{i, s i m}\right)$, where $p_{i, s i m}=1-\left(1-r_{i, s i m}\right)^{N_{i}, \operatorname{sim}}, \operatorname{logit}\left(r_{i, s i m}\right)$

$233=\alpha_{0}+\alpha_{1} X_{2, i, s i m}, X_{2, i, s i m} \sim \mathrm{N}(0,1), \alpha_{0}=-1.73$, and $\alpha_{1}=1$. Thus, an individual at an average site 234 was expected to be detected with a probability of about 0.15 per sampling occasion.

Within these scenarios, we generated false-positive detections as occurring at random

236 across all site intervals within a simulation. The probability of a false-positive detection within a

237 cell was derived such that $\theta_{f p}\left(s_{0}\right)$ constituted approximately $1 \%, 5 \%$ or $10 \%$ of $\theta_{t p}+\theta_{f p}$ with

238 random Binomial variance (absolute values of $s_{0 \text { sim }}$ ranged from $<0.001$ to roughly 0.025 ). We

239 defined $\theta_{f p}$ proportionally here rather than explicitly exploring specific values for the parameter

240 itself because few studies provide empirical estimates of false positive probabilities, and many

241 report percentages of observations that are true or false positives (e.g., Simons et al. 2007,

242 Swanson et al. 2016, Norouzzadeh et al. 2018). Furthermore, proportional definitions are also 
Generalized false positive models

243 commonly used to define thresholds for accurate data (e.g., 90\% accuracy or 95\% accuracy;

244 McShea et al. 2016, Swanson et al. 2016).

Within each scenario, we sampled positive detections at random to create the verified data $v_{i, j, s i m}($ the number of verified samples $=\{10,20,30,40,50,60,70,80,90100\}$ with 30 replicates for each level per scenario). Each of the 1800 generated datasets was used to fit both a standard RN model and a false-positive extension. We evaluated the performance of both estimators (relative bias, root squared error, standard deviation of the posterior distribution, 250 coefficient of variation, and frequentist coverage- $\%$ of $95 \%$ CI that included the true value) with respect to $\boldsymbol{\beta}, \boldsymbol{\alpha}$, and the finite sample population size $\left(\widehat{N}^{\text {tot }}\right.$, derived as $\left.\sum_{i=1}^{R} \widehat{N}_{\iota}\right)$. previously demonstrated gradients of sensitivity or precision: we expected the base estimator to exhibit more bias as the ratio of $\theta_{f p}$ to $\theta_{t p}$ increased (Clare et al. 2019), and expected the extended model to exhibit less relative bias, and estimate uncertainty with more verified samples and a 256 smaller proportion of false positives (Miller et al. 2011, Chambert et al. 2018). In practice, the 257 generating process for false positive error is probably rarely constant given that misclassification 258 probably arises from confusion between phenomena that are not randomly distributed (e.g., species). Misclassification in detection-nondetection data is essentially spatial or spatiotemporal error, and estimator sensitivity may more precisely depend upon similarity in the location or time

261 of true and false positive observations. To demonstrate this, we considered two subsequent

262 scenarios where $\beta_{0}=-1.5$ and $\beta_{1}=1$ with $\boldsymbol{\alpha}$ defined as before, and $\operatorname{logit}\left(s_{0, i}\right)=-6+\{-1,1\} X_{1, i, s i m}$

263 such that false positive observations were either negatively or positively associated with

264 abundance and the probability of true positive observations. The verification protocol was

265 simulated as previously. Empirically, simulated false positive observations constituted about $6 \%$ 
Generalized false positive models

266

267

268

269

270

271

272

273

274

275

276

277

278

279

280

281

282

283

284

285

286

287

288

of all observations, within the range of values considered previously. We fit 3 models for each of these scenarios: one in which $s_{0}$ was assumed $=0$, one in which it was assumed to be constant, and one in which it was modeled as varying in relation to $X_{1, i, s i m}$.

\section{Arrival Model}

Our exploration of the arrival model was similar. We first considered three scenarios with the following parameterization: $\operatorname{logit}\left(\psi_{i, \text { sim }}\right)=\beta_{0}+\beta_{1} X_{1, i, s i m}, X_{1, i, s i m} \sim \mathrm{N}(0,1), \beta_{0}=0$, and $\beta_{1}=0.5$; $\operatorname{logit}\left(p_{i, \text { sim }}\right)=\alpha_{0}+\alpha_{1} X_{2, i, s i m}, X_{2, i, s i m} \sim \mathrm{N}(0,1), \alpha_{0}=-2, \alpha_{1}=0.5$, and average arrival time $\varphi=$ occasion 6. True observations $y_{i, j, s i m}$ were generated as Bernoulli $\left(z_{i, s i m} \times p_{i, s i m} \times \mathrm{I}\left(a_{i, j, s i m}\right)\right)$, where $z_{i, s i m} \sim$ Bernoulli $\left(\psi_{\mathrm{i}, \mathrm{sim}}\right)$, and site and simulation specific arrival time $a_{i, j, s i m} \sim$ Poisson $(\varphi)$. That is, the occupancy probability of an average site was 0.50 and the probability that of true detection conditional on arrival and occupancy at an average site was ca. 0.12 . We simulated 300 replicates per scenario; as before, false positive detections varied as $s_{0 \text { sim }} \cong\{0.01,0.05,0.10\} \times$ $\overline{\boldsymbol{y}}_{\text {sim }}$ across scenarios, and the size of $v_{i, j, s i m}$ ranged from 10-100. We fit both the standard arrival model and the false-positive extension to each simulation replicate.

We next considered 6 additional scenarios to explore other sources of sensitivity. We defined logit $\left(s_{0, i, j, s i m}\right)=-6+\{-1,1\} X_{1, i, s i m}$ for $j>4$ and $s_{0, i, j, s i m}=0$ for $j \leq 4$, and $\alpha_{1}=\{-2,-1\}$ (at an average site, $p$ ca. 0.27 on the real scale) for four scenarios, and logit $\left(s_{0, i, j, s i m}\right)=-6+\{-1$, $1\} X_{1, i, s i m}$ for $j>2$ and $s_{0, i, j, s i m}=0$ for $j \leq 2$, and $\alpha_{1}=-2$ for two others; other values followed the previous description. While the previous three simulation scenarios considered allowed false positives to happen at random, the formulation for these might be more realistic if, for example, false positives are mostly associated to look-alike species that arrive just slightly or somewhat earlier than the focal species and have either comparable or distinct habitat associations. Empirically, these different formulations for false positive error resulted in false positives 
Generalized false positive models

289

290

291

292

293

294

295

296

297

298

299

300

301

302

303

304

305

306

307

308

309

310

accounting for roughly $6 \%\left(\alpha_{1}=-1\right.$ and $s_{0, i, j, s i m}=0$ for $\left.j \leq 4\right), 7 \%\left(\alpha_{1}=-1\right.$ and $s_{0, i, j, s i m}=0$ for $j \leq$

$2)$, and $3 \%\left(\alpha_{1}=-2\right.$ and $s_{0, i, j, s i m}=0$ for $\left.j \leq 4\right)$ of all observations. As with the RN model, we fit

models that assumed $s_{0}=0$, models that assumed $s_{0}$ was a constant, and models that (correctly)

assumed $s_{0}$ varied in relation to $\mathrm{X}_{1, \mathrm{i}, \mathrm{sim}}$. We evaluated estimator properties with respect to $\boldsymbol{\alpha}, \boldsymbol{\beta}, \hat{\varphi}$

and finite sample estimate of the proportion of occupied sites $(\widehat{P A O}$, derived for each simulation as $\left.\sum_{i=1}^{R} \widehat{z_{l}}\right)$.

\section{Evaluating transferability of $s_{0}$}

A potentially appealing property of the generalized description is that the confirmation outcomes

that primarily contribute to the estimation of $\theta_{f p}$ and the parameter's underlying generating

process are likely to be consistent regardless of how $\theta_{t p}$ is formulated. This suggests that if data

or computational resources are lacking, one might be able to use an informative prior for $\theta_{f p}$

given previous estimates of the parameter from a distinct (and more quickly fit) model. To

briefly explore transferability, we fit the original observation confirmation occupancy model

described by Chambert et al. (2015; i.e., $\theta_{t p, i}=z_{i} \times p_{i}$ ) to every simulated dataset described

previously, and then fit a model with the correct (i.e., $\mathrm{RN}$ or arrival) structure for $\theta_{t p, i}$ and for which $\theta_{f p}$ was strictly informed by a prior distribution derived from the posterior distribution of the false positive parameter estimated by the occupancy model.

\section{Application: Predicting Gray Fox Relative Abundance across Wisconsin}

The goal of the simulation study was primarily to evaluate the statistical properties of the standard and extended estimators. However, models for detection-nondetection data are also often used to predict or map in order to spatially delineate or prioritize management actions (e.g., Guélat and Kéry 2018). As a case study, we focus upon the relative abundance of gray fox 
Generalized false positive models

311 (Urocyon cinerargenteus) in Wisconsin, USA. The species has not been recently and

312 systematically monitored or surveyed across the state, and its distribution is poorly understood.

313 Here, we use data from the monitoring program Snapshot Wisconsin (Clare et al. 2019), in which

314 trail cameras are deployed by citizen scientists and classified via a crowdsourcing platform, to

315 investigate spatial patterns in fox relative abundance using the RN model. Gray foxes were

316 known to be frequently misclassified within the dataset (Clare et al. 2019 estimated the

317 probability of a single image being a false positive as $25 \%$; $95 \% \mathrm{CRI}=18 \%-33 \%$ ). We

318 modeled variation in fox expected abundance as a function of 5 environmental covariates and a

319 spatial smoothing term, with additional covariates used to explain variation in the detection

320 probability of individual foxes and the probability of false positive detections (details in

321 Appendix S3). We used indicator variable selection (Kuo and Malick 1998) to identify important

322 predictors and regularize the log-linear coefficients; we make statewide predictions by applying

323 model-averaged coefficients across a $2 \times 2 \mathrm{~km}$ lattice.

325 Carlo simulation through R v 3.4 (R Core Team 2017). Appendix S3 and Data S5 provide

326 additional details and code associated with the case study, and Appendix S4 provides code used

327 to implement the simulation study. Throughout the simulation study, we did not simulate any

328 confirmation of non-detections (i.e., simulated confirmations were randomly drawn from

329 positive observations) following Clare et al. (2019). This is because it seems inherently

330 inefficient for practitioners to wade through potentially many images or recordings of non-target

331 species within a putative period of non-detection: practically speaking, confirming 'absence' is

332 not always easy to do. Provided the likelihood for $v$ still incorporates some probability for non- 
Generalized false positive models

333 detection (even if this event is never observed), there seems to be no cost to doing so (Clare et al.

334 2019).

335 Results

336 As expected, when false positive error was essentially random, standard estimators for

337 abundance, proportion of area occupied, and arrival time were biased, and more biased when

338 false positives constituted a greater proportion of detections (Figures 2 and 3; more

339 comprehensive results in Appendix S2). Random misclassification across all time periods and

340 locations constituting 1,5 or $10 \%$ or all detections led to respective relative biases of 10,40 and

$34170 \%$ for abundance (across both simulated expected abundances of both 0.23 and 1 individual

342 per site), 3, 10 and $20 \%$ for proportion of area occupied, and 3, 20, and $40 \%$ for arrival time. The

343 extended estimators exhibited less bias and root mean squared error than the standard estimators

344 regardless of the size of the verified sample; estimator performance with regard to these metrics

345 asymptotically improved as more samples were verified, with minimal marginal improvement

346 once 25 sampling occasions were confirmed (Figures 2 and 3). The extended estimators for

347 species distribution and abundance also exhibited greater uncertainty than the standard

348 estimators, although this difference similarly shrank as more samples were verified. Uncertainty

349 patterns were reversed for arrival time (Figure 3), suggesting that false positive error was

350 inducing overdispersion in arrival relative to Poisson expectations.

351 Covariance between $\theta_{f p}$ and $\theta_{t p}$

352 The differences became more nuanced when true and false positives covaried in different

353 ways. Estimation of relative abundance, proportion of area occupied, and arrival time using the

354 extended models was unbiased or nearly unbiased regardless of how false positive error was 
Generalized false positive models

specified (less than 5\% relative bias, Figures 4, 5, and 6; Appendix S2). As with random error, extended estimator performance asymptotically improved with a larger confirmation sample. The standard estimators for population size and proportion of area occupied were more biased when false positive detections were more likely at locations where true positive detections were less likely (i.e., when false positive probability was negatively associated with a covariate itself positively associated with expected abundance or occupancy, Figures 4 and 5). Unsurprisingly, estimates of arrival time using the standard estimator were insensitive to spatial patterns in error, but were more biased as the simulated initiation of false positives occurred earlier relative to the average true arrival time (Figure 6). In fact, when false positives were simulated as starting only one sampling occasion before true positives, the standard estimator exhibited very slightly less bias and RMSE than the generating estimator (indeed, the extended estimator always exhibited some slight positive bias with regard to arrival time). This appeared to be a form of small sample bias associated with low simulated detection probability, as the standard model fit to the "true positive' simulated data was equivalent to the extended model (Appendix S2). For the standard model fit to data with simulated error, this appears to be a specific realization of offsetting biases (low detection probability offsetting some minimal difference in the start times of false and true positives).

In contrast, estimation of the proportion of area occupied became more biased as simulated false positive error started earlier (Figure 5). Moreover, occupancy estimation became more biased with when the conditional probability of truly detecting a species was lower, further indicating some potential sensitivity to small-sample bias. Arrival estimation was primarily sensitive to the conditional probability of detection insomuch as estimate uncertainty increased (Figure 6). 
Generalized false positive models

\section{Transferability of $s_{0}$}

Using an informed prior for false positive error generally resulted in parameter estimates that were strongly correlated with estimates produced when confirmation results were directly incorporated into the likelihood, particularly for the RN model (Appendix S2, Figure S1). There was more variability between point estimates of proportion of area occupied and time of arrival derived from models using an informed prior vs. incorporating the confirmation data. This did not appear to be related to the size of the verification sample; instead, relative to including confirmed observations in the likelihood, using an informed prior tended to result in slightly smaller estimates of proportion of area occupied and slightly larger estimates of arrival time (Appendix S2, Figure S2). Overall, estimator performance scarcely differed when using an informed prior rather than including confirmed locations (Appendix S2, Tables S1 - S9).

\section{Case Study}

Within our review of putative gray fox images, $67 \%$ were correctly classified; after aggregation within 179 distinct sampling occasions, $60 \%$ consisted of only true positives, and $40 \%$ consisted of only false positives (either coyote, Canis latrans or red fox, Vulpes vulpes). Both the standard and extended models estimated a negative association between fox abundance and the proportion of surrounding coniferous forest. Indicator variable selection provided less support for the inclusion of all covariates within the standard model than the extension (Appendix S3, Tables 1 and 2), and although patterns in predicted gray fox abundance were similar overall $(\mathrm{r}=0.80$ for pixel-wise point estimates, Figure 7), predictions from the standard model more strongly depict a purely spatial tend with less texture and pronounced discrepancies within the western part of Wisconsin. Although not a primary objective of this modeling exercise, we note the point estimate for expected state-wide population size using the standard model was more than $300 \%$ 
Generalized false positive models

401

402

403

404

405

406

407

408

409

410

411

412

413

414

415

416

417

418 more readily available than ever before. Ecologists are increasingly using automated recording 423 devices for sampling that allow every observation to be reviewed, and technology capable of

greater than when false positives were accounted for, although estimates overlapped substantially due to imprecision induced by the spatial terms (posterior median $=14999,95 \% \mathrm{CRI}=1982-$ $255660,80 \% \mathrm{CRI}=4754-34197$ for the model assuming only false negatives, vs. $4773,95 \%$ $\mathrm{CRI}=205-215225,80 \% \mathrm{CRI}=337-21341$; Figure 7). These differences manifested despite a trivially small estimate of the probability of a false positive detection per sampling interval (at an average site, $0.15 \%, 95 \%$ CRI $0.12 \%-0.18 \%$, Appendix S3).

\section{Discussion}

There are several potential reasons that few applied studies to date have fit models to detectionnondetection data that ignore false positive error. Investigators may not typically believe any data is misclassified or may find it difficult to collect additional data to inform model-based differentiation between true and false positive detections. These topics are not the focal area of our study, but the first is probably not often strictly true, and the second is increasingly surmountable. False positive errors have been documented within image, audio, or live observation tasks performed by individual volunteers, machine-learning algorithms, paid professionals, or via crowdsourcing (Miller et al. 2012, Swanson et al. 2016, Abra et al. 2018, Nourazaddeh et al. 2018). Like imperfect detection, false positive error may be the "rule rather than the exception" (Chen et al. 2013) unless all observations have been thoroughly reviewed (Gardiner et al. 2012).

Furthermore, as the number of described protocols for addressing false positive error continues to increase, there are fewer sampling schemes for which false positive error remains inestimable. With respect to the observation confirmation protocol, the requisite data is probably 
Generalized false positive models

424 producing verifiable data has proliferated such that confirmation may often be possible even

425 when recordings are not the primary sampling technique. Any individual with a smartphone has

426 the opportunity to produce a verifiable observation (i.e., a photograph or audio recording) out of

427 an ephemeral observation like a track, sighting, or song.

The more defensible reasons to ignore false positive error are, in our view, primarily

429 practical. These include lack of any described false positive extension for the model of interest,

437 Indeed, its extensibility exceeds our capacity to investigate it here, and we highlight potential 438 avenues for future research. More specific simulation studies are necessary to develop general rules of thumb for optimizing sampling or verification protocols (Clement 2016), particularly for model classes using binary data that were not considered here: presence-background models

441 (Renner et al. 2015), models that integrate occurrence with other data types (Chandler and Clark

442 2014, Zipkin et al. 2017), or dynamic models: our intuition is that although accounting for false

443 positive error is rarely an incorrect choice, certain models are more sensitive to false positive

444 error than others (see below). Similarly, further evaluation is needed to inform the practical 445 implementation of other protocols for false positive error (again, also extensible: Appendix S1) 
Generalized false positive models

447 within binary data $-y_{i, j} \sim$ Bernoulli $\left(\theta_{t p}+\theta_{f p}\right)$ or Bernoulli $\left(\theta_{t p}+\theta_{f p}-\left[\theta_{t p} \times \theta_{f p}\right]\right)$ - can also be

448 extended to other data types. For example, a count of organisms $y_{i, j}$ could be considered

449 distributed as Poisson $\left(\lambda_{t p}+\lambda_{f p}\right)$ assuming that each organism is counted once: developing

450 sampling protocols that enable identification of these parameters is a clear future research need.

Our simulation results demonstrate that rates of misclassification heretofore considered sufficiently accurate (e.g., $5 \%$ of classifications are false positive) can induce potentially problematic levels of bias in estimates of abundance or arrival time. However, a key point

454 emerging from our study is that intuiting whether false positive error is likely to substantively

455 influence results or not is tricky. There are two reasons. First, false positive error affects

456 estimation and inference in several varying ways. Consistent with previous work (e.g., Miller et

457 al. 2011), false positive error tended to inflate estimates of species distribution or abundance, and

458 negatively bias estimates of arrival if false positives occur prior to species arrival. One potential

459 justification for ignoring false positive error in the face of potential bias might be that the research objectives can be achieved with ordinal or proportional inference, like determining the

461 direction of a temporal trend identifying a general spatial pattern (Guillera-Arroita et al. 2015,

462 Cruickshank et al. 2019), or that the research objectives prioritize minimizing uncertainty more

463 than bias. However, we also demonstrate that false positive error can sometimes increase

464 estimate uncertainty (e.g., arrival time) and can distort predictive patterns or trends (as seen in

465 the application). Neither finding is surprising given that temporally distinct false positives are

466 likely to increase the variance in observed arrival times and that spatially distinct false positives

467 inherently alter patterns in observed occurrence or abundance. What they collectively suggest is

468 that investigators should think more carefully and broadly about how false positive error might 
Generalized false positive models

469 impact estimation of state variables or parameters of interest before assuming said error is

470 inconsequential.

Secondly, estimator sensitivity to false positive error is further dependent upon several

472 other factors. As with previous studies (e.g., Ruiz-Gutiérrez et al. 2016), we found that estimator

473 bias tended to increase when there were more false positives within the dataset. However,

474 sensitivity to false positive error is also likely to depend upon the degree of spatial or temporal

475 overlap between true and false positive observations (simulations suggest greater bias with less

476 overlap, and presumably less overlap also distorts inference regarding spatiotemporal patterns

477 more strongly), how efficiently the state process of interest is observed (distribution estimates

478 were more biased when the conditional probability of true detection was lower), and several

479 other attributes of and interactions between the state process, sampling design, and object of

480 inference. For example, the effect of false positives upon occupancy estimates is less pronounced

481 when a species is very widespread (e.g., Clare et al. 2019), and presumably, the effect of false

482 positives upon estimated arrival times depends partially upon when sampling is initiated. There

483 is also likely to be sensitivity to model form and structure. Unlike occupancy models (e.g., Clare

484 et al. 2019), the RN model exhibited consistent bias across a (limited) range of abundances.

485 Moreover, the range of potential bias for the RN model induced by false positives appears to

486 exceed the range of potential bias exhibited by occupancy models: the case study suggests that at

487 relatively high rates of false positive error, the RN model's bias can be extreme.

Other considerations include the complexity of the fitted functional response and model

489 assumptions pertaining to imperfect detection. Complex responses (e.g., splines) may be more

490 distorted by false positive error than monotonic response shapes (Fernandes et al. 2019).

491 Similarly, we expect that models assuming imperfect detection are particularly sensitive to false 
Generalized false positive models

492 positive error because species are both observed in more places, and observation patterns become

493 more heterogeneous and sparse (Kéry and Royle 2016). In contrast, a 5\% false positive rate can

494 induce at most a 5\% absolute bias in finite-sample estimates in area of occurrence assuming

495 perfect detection, and has no influence on prevalence within a presence-background model

496 because the parameter is inestimable. Given such variability that, for example, a 5\% false

497 positive rate could induce either practically no bias or $40 \%$ relative bias in estimated population

498 size, it is clear that assuming false positive error is inconsequential actually implies several

499 distinct assumptions. It is also difficult to see how general blanket definitions for data sufficiency

500 (e.g., 95\% classification accuracy) can be effective across different model types, species, or

501 sampling protocols without being extremely stringent (e.g., 99\% accuracy).

An underappreciated benefit of explicitly modeling false positive error is that it eases the

models are completely insensitive to the prevalence of error or its patterns or sampling/state

508 their false positive extensions. Still, as we have argued previously, we believe that in many cases

509 variability in estimator bias is typically muted once a model accounts for false positives (Clare et

511 univariate patterns in our simulations were accounted for, the number of confirmed samples or

512 the structure of the model for false positive error had limited influence on estimator bias. For

513 species and sampling schemes with relatively limited amounts of false positive error, there may

514 be little practical benefit to confirming more than, say, 50 observations, and a constant model for 
Generalized false positive models

515 false positive error may be sufficient (assuming the model for $\theta_{t p}$ is adequately specified)

516 because there is either limited variation to explain or limited benefit to trying to explain more

517 variation.

For species or sampling schemes with a greater prevalence of error (as was the case with the gray fox in our case study), a larger confirmation sample is likely to be necessary (Ruiz-

520 Gutiérrez et al. 2016, Chambert et al. 2018). Furthermore, as the proportion of false positive

521 error increases and there is potentially more variation in false positive error to explain, a larger

522 confirmation sample allows one to fit more complex parameterizations for false positives using

523 splines, zero-inflation terms, or functions based upon latent variables (e.g., the abundance of

524 look-alike species or the species of question). The efficacy of more complex models for false

525 positive error (and the adequacy of simpler models) across a realistic range of sampling

526 conditions is an important question. In part, we used relatively simple models for simulation

527 simply because it is not clear what constitutes a realistic or adequate generating model for false

528 positive error.

Relatedly, the degree to which accounting for false positive error reduces bias also depends upon how well the model for true positives approximates the generating process (McClintock et al. 2010b, Miller et al. 2015). In principal, the model-based solutions for false positives operate by probabilistically distinguishing true and false positives: estimation of each part of the mixture

533 is sensitive to the specification of the other. The case study represents a particularly problematic 534 dataset. The environmental associations of the gray fox were poorly understood and there was a 535 large proportion of false positive error. Even worse, this error was associated with two other generalist species with poorly delineated habitat associations that also, across much of the state,

537 are believed to exist at greater density (e.g., analysis associated with Clare et al. 2016). We 
Generalized false positive models

538 almost certainly did not fit the true models for gray fox true and false positive detections, and we

539 do not know which population estimate or predictive surface is closer to the truth. However, we

540 have higher confidence in the estimate accounting for false positive error because its predictions

541 more closely resemble the patterns described by previous survey efforts (Peterson et al. 1977)

542 and because results here and elsewhere (Miller et al. 2015) suggest that there is greater marginal

543 benefit associated with relaxing the assumption of no false positive error than marginal loss

544 associated with model misspecification.

Accounting for false-positive error adds more parameters and added dimensions to the objective function of interest-it is somewhat slower to fit extended models, and there is an

547 additional component that might be subject to model selection practices. However, we think 548 there are several ways to alleviate any additional computational burden. First, while we describe 549 the extensions using a complete data likelihood because it makes extensibility more intuitive, 550 neither the complete data likelihood nor Bayesian techniques are necessary for implementation.

551 It is also possible to integrate distinct likelihoods for $\boldsymbol{y}$ and $\boldsymbol{v}$ over possible values for any latent

552 variables to construct faster estimators using maximum likelihood or Hamiltonian-MCMC (see

553 example with the RN model in Appendix S4). Secondly, because the generating process for $\theta_{f p}$ is

554 the same regardless of how the model for $\theta_{t p}$ is specified, we think it is reasonable to try to 555 address any model selection for false positive error within a simpler model structure for $\theta_{t p}$ : for 556 example, selecting the model structure for false positives within a standard occupancy estimator.

557 Finally, defining a prior distribution for $\theta_{f p}$ informed by the estimates of a simpler model may 558 make estimation faster (if slightly less efficient) than incorporating confirmed data directly into 559 the likelihood. This is likely to produce larger computational gains when the number of 560 confirmed samples is sizable. There are, however, a few caveats. First, the confirmation data 
Generalized false positive models

561 contains information useful for inferring specific latent states, and this information is lost when

562 using a prior distribution for $\theta_{f p}$ (as it is when using a calibration protocol). Secondly, estimation

563 of $\theta_{f p}$ is likely sensitive to the specification of $\theta_{t p}$ : arrival and RN models are structurally very

564 similar to occupancy models, but transferability between occupancy models and very distinct

565 model types is less certain. Finally, it is unclear how transferable $\theta_{f p}$ is likely to be if it is

566 modeled as varying in more complex ways than considered here.

In whole, we believe we have demonstrated that it is possible and tractable to address

568 false positive error across a broader range of estimation problems than previously considered.

569 We conclude by noting that the effects of false positive error, the strategies for dealing with it,

570 and the limitations of these strategies largely mirror considerations associated with imperfect

571 detection. The importance of imperfect detection, the practicality of collecting the extra data

572 needed to estimate it within models relative to trying to control for it using design-based

573 solutions, and the stability of models accounting for imperfect detection have all been debated,

574 and these considerations are all similarly germane to false positive error. It is now generally

575 accepted that imperfect detection needs to be accounted for to achieve certain objectives and that

576 statistical techniques to do so are important ecological tools (Guillera-Arroita et al. 2015). It has

577 been argued that false positive error deserves similar wide consideration (Miller et al. 2015). The

578 generalizable model structure described here, we hope, will accelerate the adoption of techniques

579 to limit its effects while providing investigators the flexibility to fit the models they are

580 interested in fitting.

581 Acknowledgments 
Generalized false positive models

582

583

584

585

586

587

588

589

590

591

592

593

594

595

596

597

598

599

600

601

602

603

604

Support for this research was provided by NASA ESSF NNX16AO61H and NASA Ecological

Forecasting grant NNX14AC36G. We thank the associate editor and 2 anonymous reviewers for constructive comments on previous drafts.

\section{References}

Abra, F. D., M. P. Huijser, C. S. Pereira, and K. Ferraz. 2018. How reliable are your data? Verifying species identification of road-killed mammals recorded by road maintenance personnel in Sao Paulo State, Brazil. Biological Conservation 225:42-52.

Balantic, C., and T. Donovan. 2019. Dynamic wildlife occupancy models using automated acoustic monitoring data. Ecological Applications 29: e01854.

Chambert, T., D.A.W. Miller, and J. D. Nichols. 2015. Modeling false positive detections in species occurrence data under different study designs. Ecology 96:332-339.

Chambert, T., J. H. Waddle, D.A.W. Miller, S. C. Walls, and J. D. Nichols. 2018. A new framework for analyzing automated acoustic species-detection data: occupancy estimation and optimization of recordings post-processing. Methods in Ecology and Evolution 9:560-570.

Chen, G., M. Kéry, M. Plattner, K. Ma, and B. Gardner. 2013. Imperfect detection is the rule rather than exception in plant studies. Journal of Ecology 101:183-191.

Clare, J.D.J., D. W. Linden, E. M. Anderson, and D. M. MacFarland. 2016. Do the anti-predator behaviors of shared prey mediate intraguild predation and mesopredator suppression? Ecology and Evolution 6:3884-3897.

Clare, J.D.J., P. A. Townsend, C. Anhalt-Depies, C. Locke...et al. 2019. Making inference with messy (citizen science) data: when are data accurate enough and how can they be improved. Ecological Application 29: e01849. 
Generalized false positive models

605

606

607

608

609

610

611

612

613

614

615

616

617

618

619

620

621

622

623

624

625

626

627

Clement, M. J. 2016. Designing occupancy studies when false-positive detections occur. Methods in Ecology and Evolution 7:1538-1546.

Cruickshank, S. S., C. Buhler, and B. R. Schmidt. 2019. Quantifying data quality in a citizen science monitoring program: false negatives, false positives, and occupancy trends. Conservation Science and Practice 1: e54.

Gardiner, M. M., L. L. Allee, P. M. Brown, J. E. Losey, H. E. Roy, and R. R. Smyth. 2012. Lessons from lady beetles: accuracy of monitoring data from US and UK citizen-science programs. Frontiers in Ecology and the Environment 10:471-476.

Guillera-Arroita, G., J. J. Lahoz-Monfort, J. Elith, A. Gordon, H. Kujala, P. E. Lentini, M.A. McCarthy, R. Tingley, and B. A. Wintle. 2015. Is my species distribution model fit for purpose? Matching data and models to applications. Global Ecology and Biogeography 24:276-292.

Guélat, J. and Kéry, M. 2018. Effects of spatial autocorrelation and imperfect detection on species distribution models. Methods in Ecology and Evolution 9:1614-1625.

Ferguson, P.F.B., M. J. Conroy, and J. Heppinstall-Cymerman. 2015. Occupancy models for data with false positive and false negative errors and heterogeneity across sites and surveys. Methods in Ecology and Evolution 6:1395-1406.

Fernandes, R. F., D. Scherrer, and A. Guisan. 2019. Effects of simulated observation errors on the performance of species distribution models. Diversity and Distribution 25:400-413.

Kéry, M., and J. A. Royle. 2016. Applied hierarchical modeling in ecology, Volume 1. Academic Press, London.

Kosmala, M., A. Wiggins, A. Swanson, and B. Simmons. 2016. Assessing data quality in citizen science. Frontiers in Ecology and the Environment 14:551-560. 
Generalized false positive models

628

629

630

631

632

633

634

635

636

637

638

639

640

641

642

643

644

645

646

647

648

MacKenzie, D. I., J. D. Nichols, G. B. Lachman, S. Droege, J. A. Royle, and C. A. Langtimm. 2002. Estimating site occupancy rates when detection probabilities are less than one. Ecology 83:2248-2255.

McClintock, B. T., L. L. Bailey, K. H. Pollock, and T. R. Simons. 2010a. Experimental investigation of observation error in anuran call surveys. Journal of Wildlife Management $74: 1882-1893$.

McClintock, B.T., Bailey, L.L., Pollock, K.H.\& Simons, T.R. 2010b. Unmodeled observation error induces bias when inferring patterns and dynamics of species occurrence via aural detections. Ecology, 91, 2446-2454.

McShea, W. J., T. Forrester, R. Costello, Z. He, and R. Kays. 2016. Volunteer-run cameras as distributed sensors for macrosystem mammal research. Landscape Ecology 31:55-66.

Miller, D. A., J. D. Nichols, B. T. McClintock, E. H. Campbell Grant, L. L. Bailey, and L. A. Weir. 2011. Improving occupancy estimation when two types of observational error occur: non-detection and species misidentification. Ecology 92:1422-1428.

Miller, D. A. W., L. A. Weir, B. T. McClintock, E. H. Cambell Grant, L. L. Bailey, and T. R. Simons. 2012. Experimental investigation of false positive errors in auditory species occurrence surveys. Ecological Applications 22:1665-1674.

Miller, D.A.W., L. L. Bailey, E. H. C. Grant, B. T. McClintock, L. A. Weir, and T. R. Simons. 2015. Performance of species occurrence estimators when basic assumptions are not met: a test using field data where true occupancy status is known. Methods in Ecology and Evolution 6:557-565. 
Generalized false positive models

Miller, D. A. W., K. Pacific, J. S. Sanderlin, and B. J. Reich. 2019. The recent past and promising future for data integration methods to estimate species' distributions. Methods in Ecology and Evolution 10:22-37.

Norouzzadeh, M. S., A. Nguyen, M. Kosmala, A. Swanson, M. S. Palmer, C. Packer, and J. Clune. 2018. Automatically identifying, counting, and describing wildlife animals in camera-trap images with deep learning. Proceedings of the National Academy of Sciences: 201719367.

Otis, D. L., K. P. Burnham, G. C. White, and D. R. Anderson. 1978. Statistical inference from capture data on closed animal populations. Wildlife Monographs 62:3-135.

Petersen, L. R., M. A. Martin, and C. M. Pils. 1977. Status of gray foxes in Wisconsin. Wisconsin Department of Natural Resources Resource Report 94, Madison, USA.

Plummer, M. (2003). JAGS: a program for analysis of Bayesian gra $\varphi$ cal models using GIBBS sampling. Proceedings of the $3^{\text {rd }}$ international workshop on distributed statistical computing.

Swanson, A., M. Kosmala, C. Lintott, and C. Packer. 2016. A generalized approach for producing, quantifying, and validating citizen science data from wildlife images. Conservation Biology 30:520-531.

R Core Team. 2017. R: a language and environment for statistical computing. R Foundation for Statistical Computing, Vienna.

Ramsey, D. S. L., P. A. Caley, and A. Robley. 2015. Estimating population density from presence-absence data using a spatially explicit model. Journal of Wildlife Management 79:491-499.

Renner, I. W., J. Elith, A. Baddely, W. Fithian, T. Hastie, S. J. Phillips, G. Popovic, and D. I. 
Generalized false positive models

672

673

674

675

676

677

678

679

680

681

682

683

684

685

686

687

688

689

690

691

692

693

Warton. 2015. Point process models for presence-only analysis. Methods in Ecology and Evolution 6:366-379.

Rossman, S., C. Yackulic, S. Saunders, J. Reid, and E. F. Zipkin. 2016. Dynamic N-occupancy models: estimating demographic rates and local abundance from detection-nondetection data. Ecology 97:3300-3307.

Roth, T., N. Strebel, and V. Amrhein. 2014. Estimating unbiased phenological trends by adapting site-occupancy models. Ecology 95:2144-2154.

Royle, J. A., and W. A. Link. 2006. Generalized site occupancy models allowing for false positive and false negative errors. Ecology 87:835-841.

Royle, J. A., and J. D. Nichols. 2003. Estimating abundance from repeated presence-absence data or point counts. Ecology 84:777-790.

Ruiz-Gutiérrez, V., M. B. Hooten, and E. H. Campbell Grant. 2016. Uncertainty in biological monitoring: a framework for data collection and analysis to account for multiple sources of sampling bias. Methods in Ecology and Evolution 7:900-909.

Simons, T. R., M.W. Alldredge, K. H. Pollock, and J. M. Wettroth. 2007. Experimental analysis of the auditory detection process on avian point counts. Auk 124:986-999.

Swanson, A., M. Kosmala, C. Lintott, and C. Packer. 2016. A generalized approach for producing, quantifying, and validating citizen science data from wildlife images. Conservation Biology 30:520-531.

Zipkin, E. F., S. Rossman, C. B. Yackulic, J. D. Wiens, J. T. Thorson, R. J. Davis, and E. H. Cambell Grant. 2017. Integrating count and detection-nondetection data to model population dynamics. Ecology 98:1640-1650. 
Generalized false positive models

Figure 1. Schematic for the generalized observation-confirmation model for false positive error presented here. Unconfirmed outcomes are positive if the species has either been truly or falsely detected, or negative if neither true nor false detection has occurred. Confirmed data can either result in a true detection only, a false detection only, both, or no detection. The probability of $>0$ false detections within a verified sampling interval is denoted $\theta_{f p}$, while the probability of $>0$ true detections, $\theta_{t p, i}$, is treated as a combination of functions associated with state and conditional observation models.

Figure 2. Performance of the standard Royle-Nichols model (when 0 observations are confirmed) and the model extension for false positive error with regard to finite-sample population size under varying levels of random false positive error $(\%$ of total observations $=1$, 5, or 10) and confirmation effort. $\mathrm{SE}=$ standard deviation of the posterior distribution, $\mathrm{CV}=$ coefficient of variation.

Figure 3. Performance of a standard phenological occupancy model (when 0 observations are confirmed) and the model extension for false positive error with regard to proportion of area occupied and the time of arrival under varying levels of random false positive error (\% of total observations $=1,5$, or 10 ) and confirmation effort. $\mathrm{SE}=$ standard deviation of the posterior distribution, $\mathrm{CV}=$ coefficient of variation.

Figure 4. Performance of the standard Royle-Nichols model (when 0 observations are confirmed) and the model extension for false positive error with regard to finite-sample population size under different directional covariance between true and false positive detections, and when different models for false positive error (either a misspecified constant model or the generating model) were fit. $\mathrm{SE}=$ standard deviation of the posterior distribution, $\mathrm{CV}=$ coefficient of variation. 
Generalized false positive models

Figure 5. Performance of the standard standard phenological occupancy model (when 0 observations are confirmed) and the model extension for false positive error with finite-sample population size with regard to proportion of area occupied under different directional covariance between true and false positive detections, and when different models for false positive error (either a misspecified constant model or the generating model) were fit. SE = standard deviation of the posterior distribution, $\mathrm{CV}=$ coefficient of variation.

Figure 6. Performance of the standard standard phenological occupancy model (when 0 observations are confirmed) and the model extension for false positive error with finite-sample population size with regard to estimated arrival time under different directional covariance between true and false positive detections, and when different models for false positive error (either a misspecified constant model or the generating model were fit). SE = standard deviation of the posterior distribution, $\mathrm{CV}=$ coefficient of variation.

Figure 7. Predictions of gray fox relative abundance across Wisconsin, USA in 2017 using the standard Royle-Nichols estimator (A) and the extended version of the estimator (B) exhibit differences in pattern and granularity; posterior distributions of expected statewide abundance truncated at the upper 95\% CI (C) and at 50000 (D) for visualization purposes. 
Generalized false positive models

A) Confirmed Data

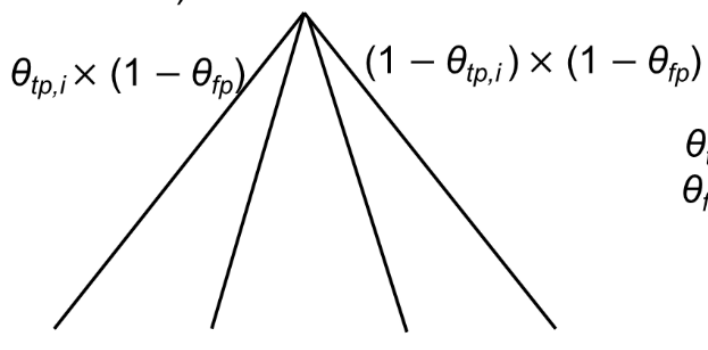

$v_{i, j}=1 \quad v_{i, j}=2 \quad v_{i, j}=3 \quad v_{i, j}=4$

$$
\theta_{f p} \times\left(1-\theta_{t p, i}\right) \quad \theta_{t p, i} \times \theta_{f p}
$$

$\boldsymbol{\theta}_{t p}=$ Unconditional probability of (true) detection at a location during a given

sampling interval $=$ combination of state and observation processes

$\theta_{f p}=s_{0}=$ Probability of false detection within a sampling interval

$\boldsymbol{\Omega}_{\mathbf{i}}=\left[\left\{\left(s_{1, i} \times\left(1-s_{0}\right)\right\}\left\{s_{0} \times\left(1-s_{1, i}\right)\right\}\left\{s_{1, i} \times s_{0}\right\}\left\{\left(1-s_{1, i}\right) \times\left(1-s_{0}\right)\right\}\right]\right.$

$$
\begin{gathered}
v_{i, j} \sim \text { Categorical }\left(\mathbf{\Omega}_{\mathrm{i}, \mathrm{j}}\right) \\
y_{i, j} \sim \text { Bernoulli }\left(1-\Omega_{4, \mathrm{i}, \mathrm{j}}\right) \\
\operatorname{Pr}\left(y_{i, j}=0\right)=\operatorname{Pr}\left(v_{i, j}=4\right) \\
\operatorname{Pr}\left(y_{i, j}=1\right)=\operatorname{Pr}\left(v_{i, j} \neq 4\right)
\end{gathered}
$$

Occupancy Model: $\theta_{t p, i}=z_{i} \times p$

Royle-Nichols Model: $\theta_{t p, i}=1-(1-r)^{N_{i}}$

Spatial Royle-Nichols Model: $\theta_{t p, j}=1-\prod_{i=1}^{N}\left(1-\left(g_{0} \times \frac{-d_{i, j}}{2 \sigma^{2}} \times z_{i}\right)\right)$

Arrival Model: $\theta_{t p, i, j}=z_{i} \times p \times I\left(j>x_{i}\right)$

\section{Figure 1.}


Generalized false positive models
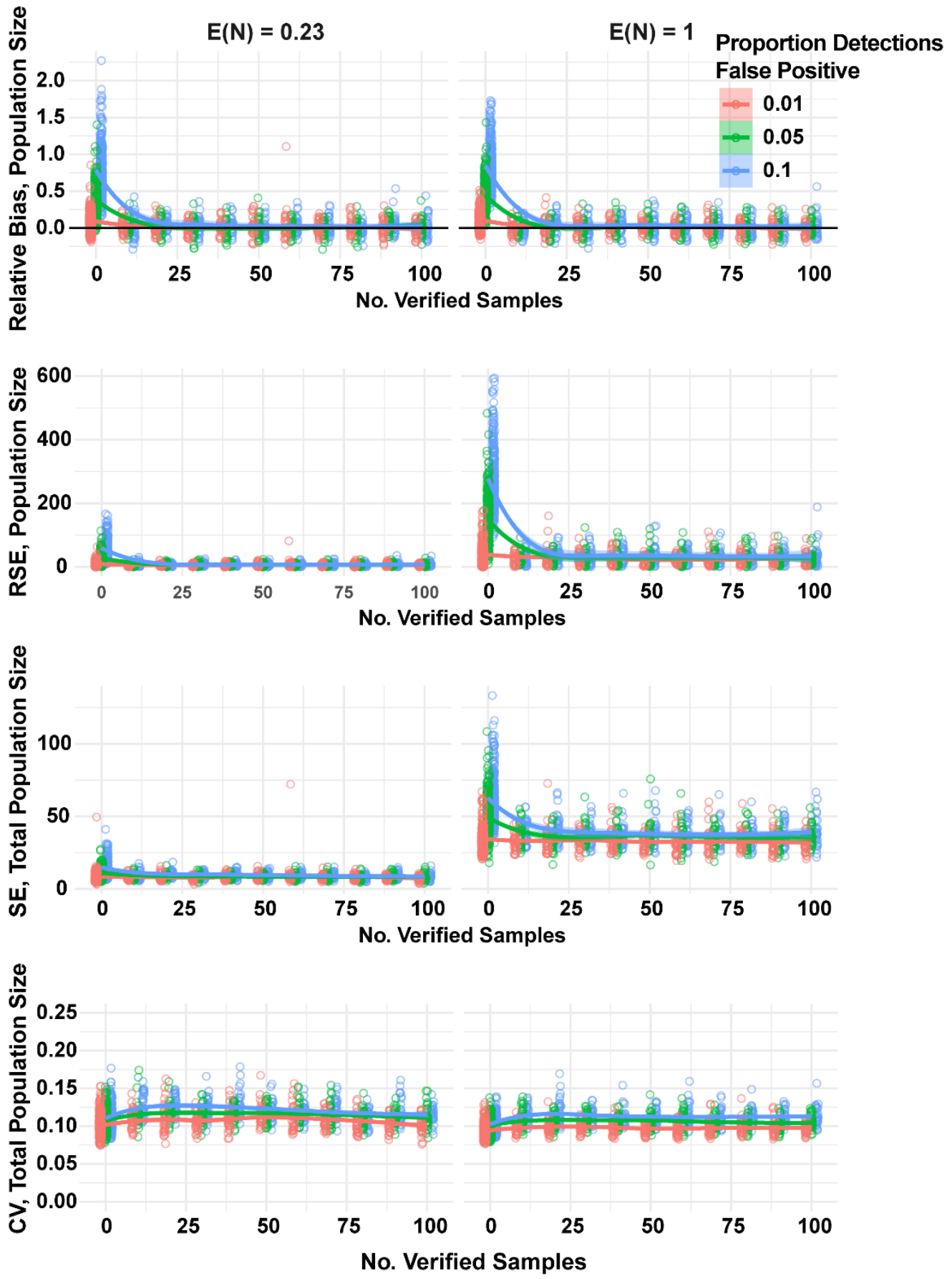

Figure 2. 
Generalized false positive models
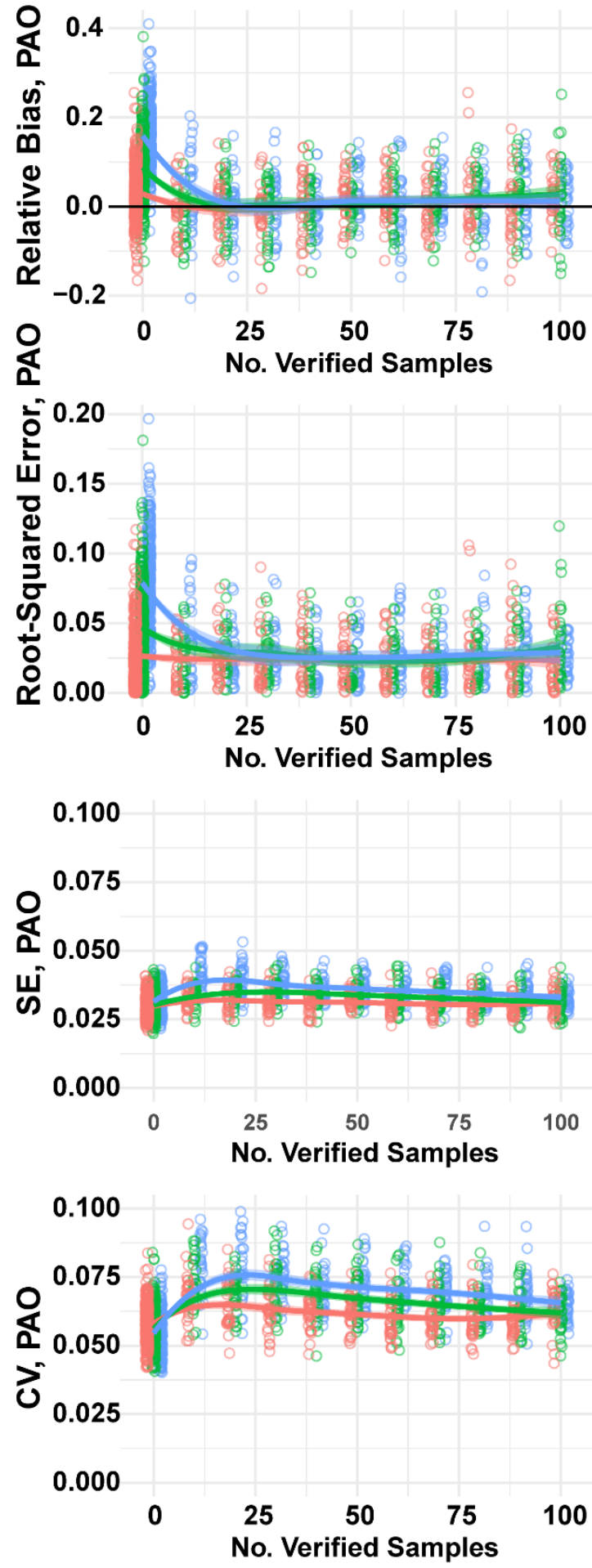
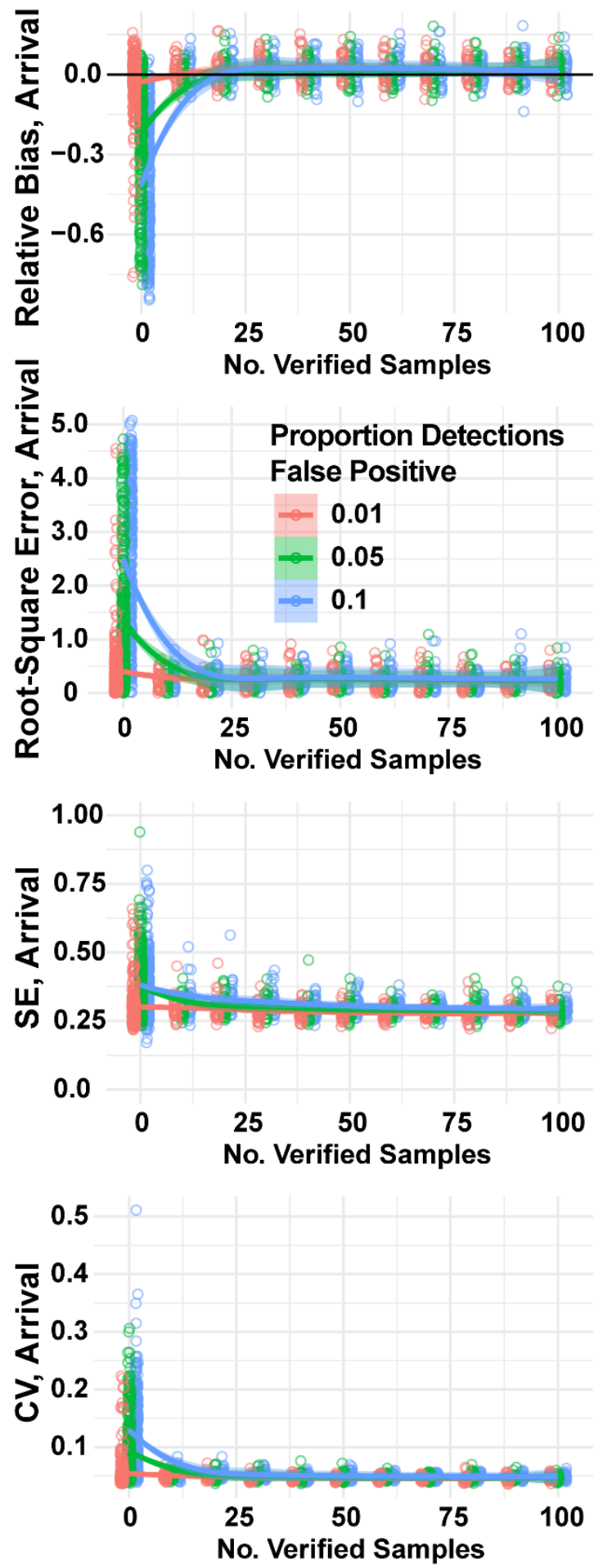

Figure 3. 
bioRxiv preprint doi: https://doi org/10.1101/422527; this version posted September 17, 2019. The copyright holder for this preprint (which was not certified by peer review) is the author/funder, who has granted bioRxiv a license to display the preprint in perpetuity. It is made available under aCC-BY-NC-ND 4.0 International license.

Generalized false positive models

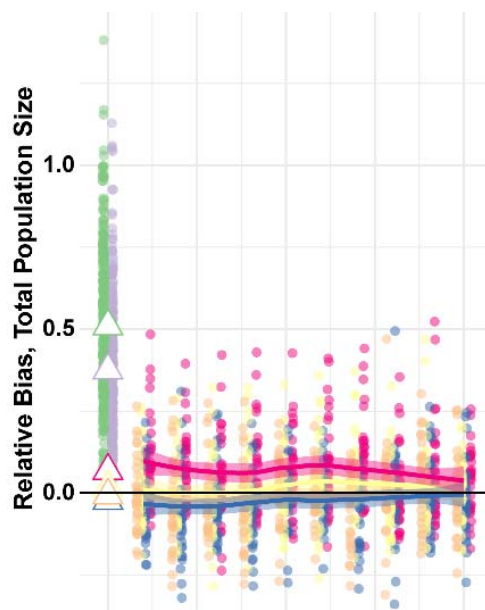

0
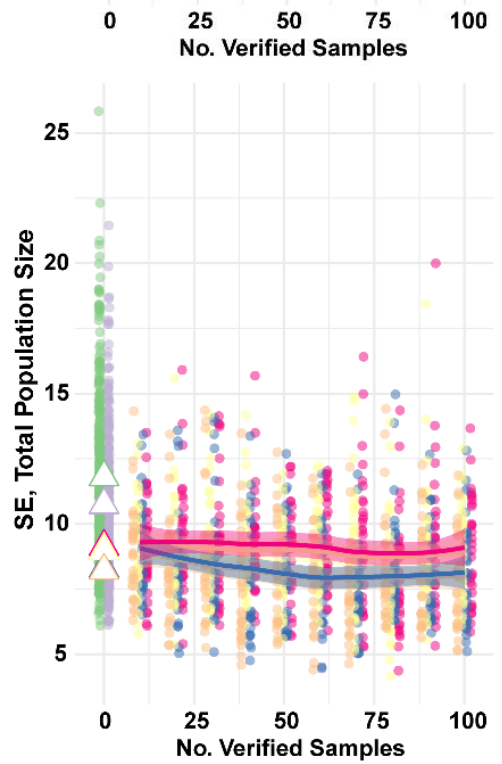
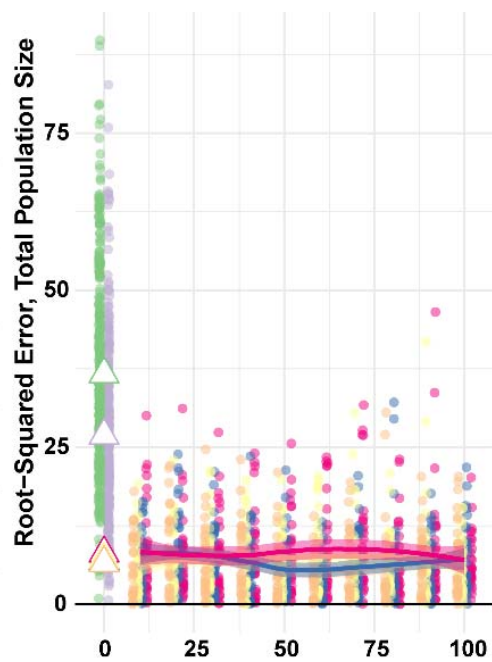
No. Verified Samples

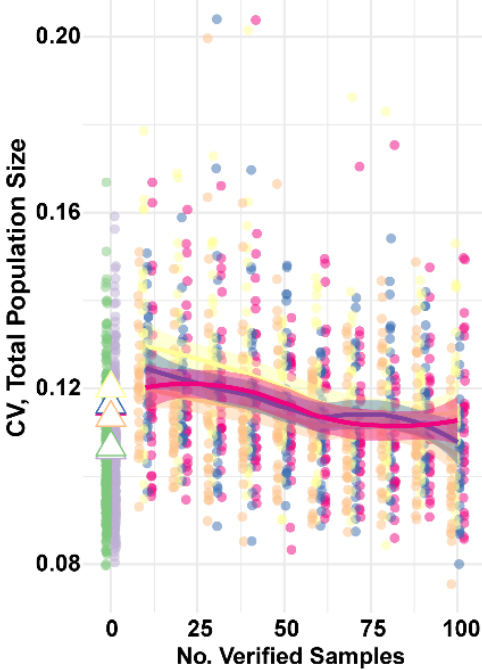

Negative Covariance, Base Model

Positive Covariance, Base Model

Negative Covariance, FP w/ Covariate

Positive Covariance, FP w/ Covariate

- Negative Covariance, FP, No Covariate

- Positive Covariance, FP, No Covariate

$\triangle$ Mean Values Accross all Verification Levels

\section{Figure 4.}


bioRxiv preprint doi: https://doi.org/10.1101/422527; this version posted September 17 , 2019. The copyright holder for this preprint (which was not certified by peer review) is the author/funder, who has granted bioRxiv a license to display the preprint in perpetuity. It is made available under aCC-BY-NC-ND 4.0 International license.

Generalized false positive models
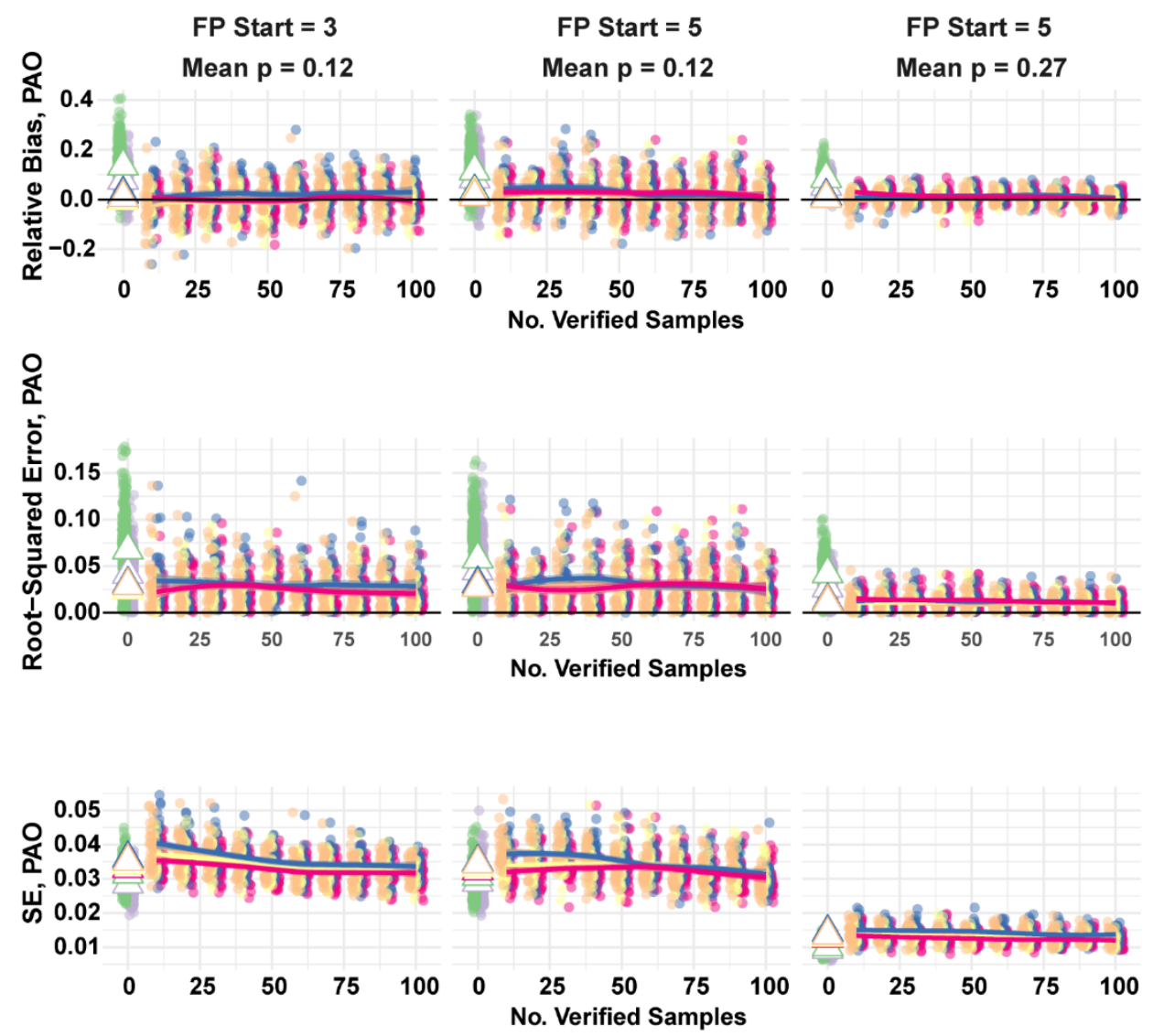

\section{Negative Covariance, Base Model \\ Positive Covariance, Base Model}

Negative Covariance, FP w/ Covariate

Positive Covariance, FP wl Covariate

스 Negative Covariance, FP, No Covariate

Positive Covariance, FP, No Covariate

$\triangle$ Mean Values Accross all Verification Levels

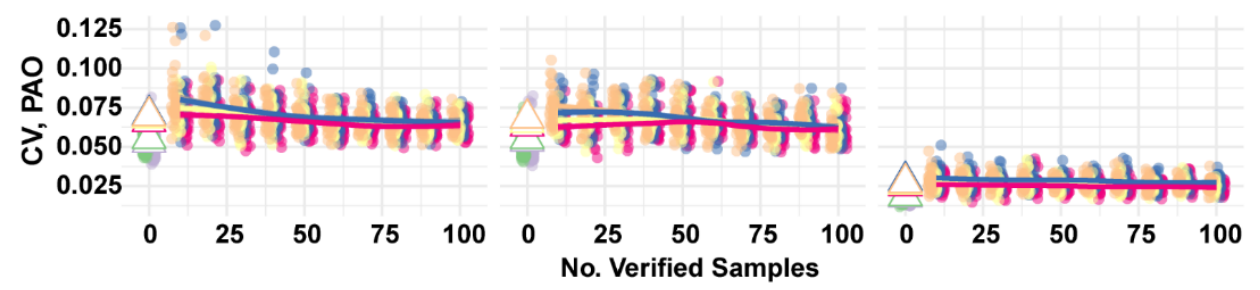

Figure 5. 
bioRxiv preprint doi: https://doi.org/10.1101/422527; this version posted September 17 , 2019. The copyright holder for this preprint (which was not certified by peer review) is the author/funder, who has granted bioRxiv a license to display the preprint in perpetuity. It is made available under aCC-BY-NC-ND 4.0 International license.

Generalized false positive models
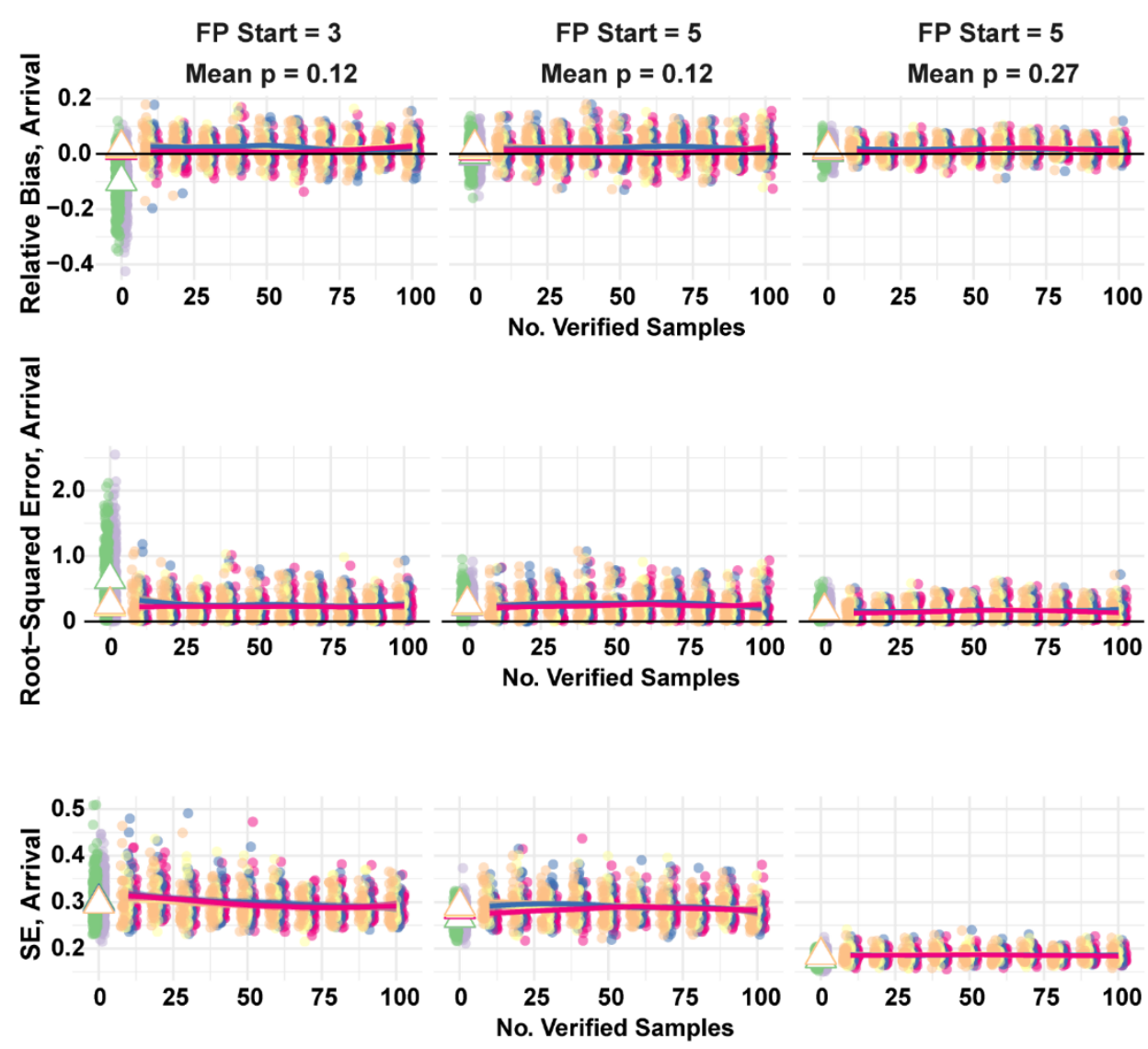

Negative Covariance, Base Model

Positive Covariance, Base Model

Negative Covariance, FP wl Covariate

Positive Covariance, FP w/ Covariate

Negative Covariance, FP, No Covariate

- Positive Covariance, FP, No Covariate

$\triangle$ Mean Values Accross all Verification Levels

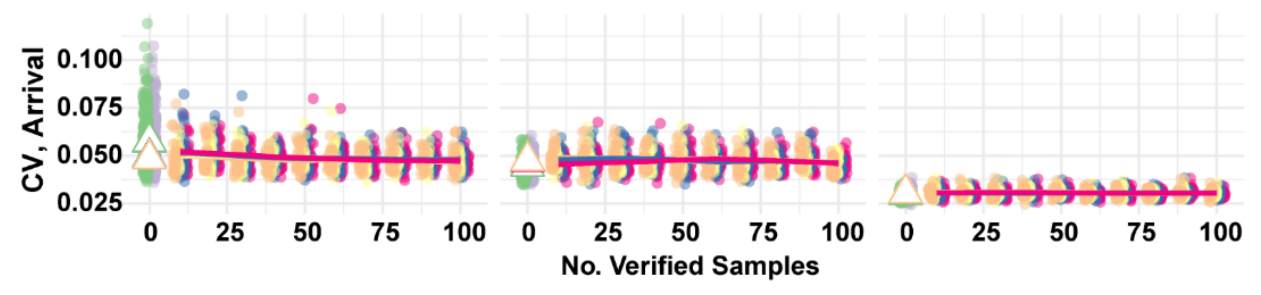

\section{Figure 6.}


bioRxiv preprint doi: https://doi org/10.1101/422527; this version posted September 17, 2019. The copyright holder for this preprint (which was not certified by peer review) is the author/funder, who has granted bioRxiv a license to display the preprint in perpetuity. It is made available under aCC-BY-NC-ND 4.0 International license.

Generalized false positive models

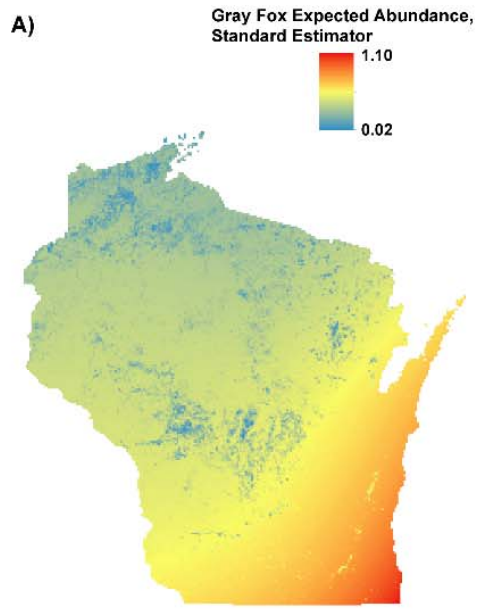

\section{B)

(a)

Gray Fox Expected Abundance,

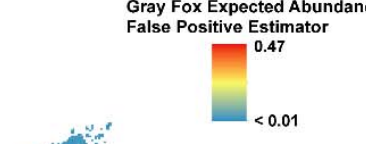

C)

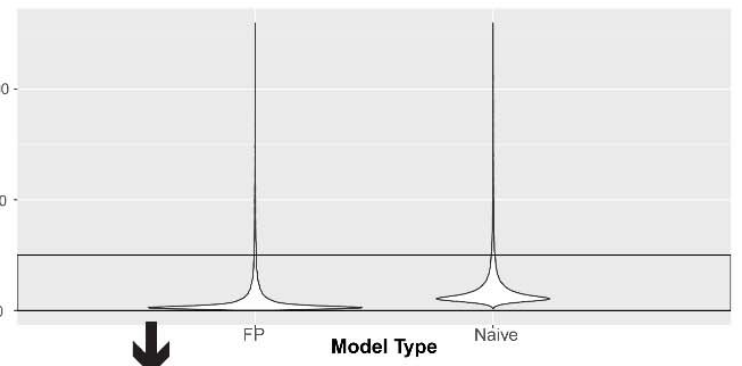

D)

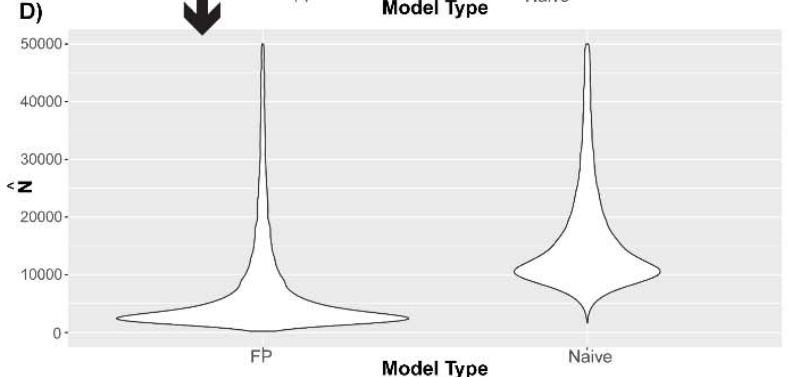

\section{Figure 7.}


Generalized false positive models

\section{Appendix S1. Other model extensions.}

Extension of other false-positive models.

Despite our focus on leveraging observation-confirmation to deal with false positive error, it is important to note that other strategies for estimating false positive parameters can also be effective across different model types. We describe here alternative formulations following different described protocols for dealing with false positive error when estimating relative abundance using the model of Royle and Nichols (2003). The base model can be expressed hierarchically as:

$$
\begin{gathered}
N_{i} \sim \text { Poisson }(\lambda) \\
p_{i}=1-(1-r)^{N_{i}} \\
y_{i, j} \sim \operatorname{Bernoulli}\left(p_{i}\right)
\end{gathered}
$$

The description for the observation-confirmation extension described in the main text is:

$$
\begin{gathered}
N_{i} \sim \text { Poisson }(\lambda) \\
\theta_{t p, i}=1-(1-r)^{N_{i}} \\
\theta_{f p}=\mathrm{s}_{0} \\
\mathbf{\Omega}_{\boldsymbol{i}}=\left[\left\{\theta_{t p, i} \times\left(1-\theta_{f p}\right)\right\}\left\{\theta_{f p} \times\left(1-\theta_{t p, i}\right)\right\}\left\{\theta_{f p} \times \theta_{t p, i}\right\}\left\{\left(1-\theta_{t p, i}\right) \times\left(1-\theta_{f p}\right)\right\}\right] \\
v_{i, j} \sim \text { Categorical }\left(\mathbf{\Omega}_{i}\right) \\
y_{i, j} \sim \text { Bernoulli }\left(1-\Omega_{4, i}\right)
\end{gathered}
$$

As noted in the main text, a distinctive feature of the observation-confirmation protocol is that true and false positives are not necessarily mutually exclusive in space or space/time. The 
Generalized false positive models

alternative protocols do require the assumption that true and false positive observations are distinct in space (i.e., false positive observations can only occur in places where true positive observations are impossible. This may not reflect the reality of the sampling itself (i.e., there may well be true and false positive observations at the same location or the same location at the same time), but is a constraint imposed by the nature of the information that these protocols make available. As noted in the discussion, we do not know how effectively the other protocols extend to other model structures...this is an open research question that deserves attention.

For the RN model, perhaps the most logical way to make $\theta_{f p}$ and $\theta_{t p}$ mutually exclusive is to constrain $\theta_{f p}$ to only occur at locations where abundance is zero and true positives cannot occur. A formulation in the spirit of Royle and Link (2006) follows:

$$
\begin{gathered}
N_{i} \sim \text { Poisson }(\lambda) \\
\theta_{t p, i}=1-\left(1-r_{l}\right)^{N_{i}} \\
\theta_{f p, i}=I\left(N_{i}=0\right) \times r_{0} \\
r_{0}<r_{l} \\
y_{i, j} \sim \operatorname{Bernoulli}\left(\theta_{t p, i}+\theta_{f p, i}\right)
\end{gathered}
$$

Here, $I\left(N_{i}=0\right)$ denotes an indicator function for whether the abundance of site $i=0$, and $r_{0}$ denotes the probability of falsely detecting an organism at a location with zero abundance. The constraint $r_{0}<r_{l}$, where $r_{l}$ is the probability of detecting a single present organism, mirrors the constraint in the original model that $p_{10}<p_{11}$.

Within the site-confirmation protocol (e.g., Chambert et al. 2015), the observed observations $y_{i, j}$ follow a categorical distribution pertaining to whether no species was observed, 
Generalized false positive models

a species was observed but not confirmed, and a species was unambiguously observed. The parameter $b$ describes the conditional probability that a positive observation is unambiguous. A Royle-Nichols variant of the site-confirmation protocol can be described as:

$$
\begin{gathered}
N_{i} \sim \operatorname{Poisson}(\lambda) \\
\theta_{t p, i}=1-\left(1-r_{l}\right)^{N_{i}} \\
\theta_{f p, i}=I\left(N_{i}=0\right) \times r_{0} \\
\mathbf{\Omega}_{\mathbf{j}}=\left[\left\{\left(1-\theta_{t p, j}\right) \times\left(1-\theta_{f p, i}\right)\right\}\left\{\left(\theta_{t p, j} \times(1-b) \times\left(1-\theta_{f p, i}\right)\right)+\left(1-\theta_{t p, j}\right) \times \theta_{f p, i}\right)\right\}\left\{\theta_{t p, j} \times b \times(1-\right. \\
\left.\left.\left.\theta_{f p, i}\right)\right\}\right] \\
y_{i, j} \sim \text { Categorical }\left(\mathbf{\Omega}_{\mathbf{j}}\right)
\end{gathered}
$$

One variant of the site-confirmation protocol, the multiple detection methods model (Miller et al. 2011, Chambert et al. 2015), assumes that one sampling method (M1) generates ambiguous positive detections $y_{i, j}$ and a second independent method (M2) operating during different occasions $s$ always provides unambiguous positive detections $w_{i, s}$. The distinct probabilities of truly detecting a single animal for M1 and M2 can respectively be described as $r_{1}$ and $r_{2}$. The hierarchical formulation is then:

$$
\begin{gathered}
N_{i} \sim \text { Poisson }(\lambda) \\
\theta_{t p, i, 1}=1-\left(1-r_{1}\right)^{N_{i}} \\
\theta_{t p, i, 2}=1-\left(1-r_{2}\right)^{N_{i}} \\
\theta_{f p, i}=I\left(N_{i}=0\right) \times r_{0}
\end{gathered}
$$


Generalized false positive models

$$
\begin{gathered}
y_{i, j} \sim \operatorname{Bernoulli}\left(\theta_{t p, i, 1+} \theta_{f p, i}\right) \\
w_{i, s} \sim \operatorname{Bernoulli}\left(\theta_{t p, i, 2}\right)
\end{gathered}
$$

Finally, following the calibration protocol (Chambert et al. 2015), an investigator might have collected reference detection data under experimental conditions in which the state is known (or at least, the exclusive conditions for $\theta_{t p}$ and $\theta_{f p}$ are known): $x_{1}$ and $x_{0}$ respectively denote the total number of true and false positive detections in the reference data set, and $n_{1}$ and $n_{0}$ the number of trials in which true positive detections were possible or not-an example trial might be the presentation of a single image or recording in which the species of interest is present or not present. One way to specify the Royle-Nichols model hierarchically following the calibration protocol is:

$$
\begin{gathered}
N_{i} \sim \text { Poisson }(\lambda) \\
\theta_{t p, i}=1-\left(1-r_{l}\right)^{N_{i}} \\
\theta_{f p, i}=I\left(N_{i}=0\right) \times r_{0} \\
x_{1} \sim \operatorname{Binomial}\left(n_{1}, r_{l}\right) \\
x_{0} \sim \operatorname{Binomial}\left(n_{0}, r_{0}\right) \\
y_{i, j} \sim \operatorname{Bernoulli}\left(\theta_{t p, i}+\theta_{f p, i}\right)
\end{gathered}
$$

The key to implementing these different protocols within different model structures is only slightly more complex than extending the observation-confirmation protocol: one must redefine $\theta_{t p, i}$ following the original model description, and redefine $\theta_{f p, i}$ so that is exclusive of $\theta_{t p, i}$, and alter the statements associated with the auxiliary data appropriately. For example, to 
Generalized false positive models

define extensions for the arrival model following the protocols above instead of the RN model, define $\theta_{t p, i, j}=z_{i} \times p_{1} \times \mathrm{I}\left(j>x_{i}\right), \theta_{f p, i, j}=\left(1-\left(z_{i} \times \mathrm{I}\left(j>x_{i}\right)\right) \times p_{0}\right.$, and throughout, replace $r_{0}, r_{l}$, and $r_{2}$ with, respectively, (say) $p_{0}, p_{1}$, and $p_{2}$.

\section{Observation Confirmation Protocol for the Spatial Royle-Nichols Model}

The spatial Royle-Nichols model (Ramsay et al. 2015) uses $z_{i}$ to denote whether individuals $i=$ $1,2 \ldots \mathrm{M}$ exist within a geographic space $\|S\|$ with probability $\psi$. The state variable of interest, population size $N$ in $\|S\|$, is estimated as $\widehat{N}=\sum_{i=1}^{M} z_{i}$, and population density is derived as $\widehat{N} /$ Area $_{\|S\|}$. Individuals have distinct activity centers located within $\|S\|$ and the coordinates of these activity centers are denoted as $s_{i}$; individuals are detected at any of $j$ detectors on given sampling occasions $k$ with probability $p_{i, j}$. The unconditional probability of detection is a function of whether an individual exists, the distance between an individual's latent activity center and the location of the detector, $d_{i, j}$, and the parameters $g_{0}$ and $\sigma$ that respectively relate to the probability of individual detection at $d_{i, j}=0$ and the rate at which individual encounter probability decays, and can be expressed as $p_{i, j}=g_{0}\left(-d_{i, j} / 2 \sigma^{2}\right) \times z_{i}$. Individuals are not distinguished, so these parameters are inferred by marginalizing across the latent individual encounter histories at a specific detector such that the unconditional probability of detection is described as $\theta_{t p, j}=1-\prod_{i=1}^{M}\left(1-p_{i, j}\right)$. The hierarchical likelihood is:

$$
\begin{gathered}
z_{i} \sim \operatorname{Bernoulli}(\psi) \\
p_{i, j}=g_{0}\left(-d_{i, j} / 2 \sigma^{2}\right) \times z_{i} \\
\theta_{t p, j}=1-\prod_{i=1}^{M}\left(1-p_{i, j}\right) \\
\theta_{f p}=s_{0}
\end{gathered}
$$


Generalized false positive models

$$
\mathbf{\Omega}_{\mathbf{j}}=\left[\left\{\theta_{t p, j} \times\left(1-\theta_{f p}\right)\right\}\left\{\theta_{f p} \times\left(1-\theta_{t p, j}\right)\right\}\left\{\theta_{f p} \times \theta_{t p, j}\right\}\left\{\left(1-\theta_{t p, j}\right) \times\left(1-\theta_{f p}\right)\right\}\right]
$$

Here, $v_{j, k} \sim$ Categorical $\left(\mathbf{\Omega}_{\mathbf{j}}\right)$ and $y_{j, k} \sim$ Bernoulli $\left(1-\Omega_{4, j}\right)$. Critically, $\theta_{t p, j}$ has no support at 0 , and as a result, without re-specifying the SRN model or one of the false positive protocols, siteconfirmation or calibration approaches to false positive error are impossible to implement. Although not directly considered within the manuscript, code required to simulate and fit extended SRN models is found in within Appendix S4. As a general proof of concept that the model is sensitive to false positive error and an extended version can reduce bias, we present very limited simulation results here.

We simulated 100 replicate datasets to demonstrate proof of concept. Sampling parameters included a population size of 50 organisms; $\|S\|$ defined as a $20 \times 20$ unit square; detection parameters $g_{0}=0.15$ and $\sigma=0.5 ; 196$ detector locations within a $14 \times 14$ square grid with 1 unit spacing, and 20 sampling intervals: only the location of individual activity centers varied across simulation replicates. False positive observations (as 10\% of all detections) and a verification sample were simulated following practices described in the main text; the size of the verification sample varied as\{10203040 50\} replicated 20 times each. We compared the standard model and the false positive extension on the basis of relative bias of $\widehat{N}$. The standard estimator was strongly biased (relative bias $=0.81$ ) at a $10 \%$ false positive rate; the extended model was not unbiased-perhaps due to small sample size considerations—-but exhibited better performance (relative bias $=0.25$ ). Clearly more research is needed to understand the general sensitivity of the SRN model to false positive error. Given the narrow range of parameter space within which the base model is unbiased (Ramsey et al. 2015), we expect that it is more likely to see usage as a component within integrated models (Sun et al. 2019), and we suggest that it may be more fruitful to explore sensitivity to false positives within this class of model. 
Generalized false positive models

\section{References}

Chambert, T., D.A.W. Miller, and J. D. Nichols. 2015. Modeling false positive detections in species occurrence data under different study designs. Ecology 96:332-339.

Miller, D. A., J. D. Nichols, B. T. McClintock, E. H. Campbell Grant, L. L. Bailey, and L. A. Weir. 2011. Improving occupancy estimation when two types of observational error occur: non-detection and species misidentification. Ecology 92:1422-1428.

Ramsey, D. S. L., P. A. Caley, and A. Robley. 2015. Estimating population density from presence-absence data using a spatially explicit model. Journal of Wildlife Management 79:491-499.

Roth, T., N. Strebel, and V. Amrhein. 2014. Estimating unbiased phenological trends by adapting site-occupancy models. Ecology 95:2144-2154.

Royle, J. A., and J. D. Nichols. 2003. Estimating abundance from repeated presence-absence data or point counts. Ecology 84:777-790.

Royle, J. A., and W. A. Link. 2006. Generalized site occupancy models allowing for false positive and false negative errors. Ecology 87:835-841.

Sun, C. C., A. K. Fuller, and J. A. Royle. 2019. Incorporating citizen science data in spatially explicit integrated population models. Ecology:e027777. 
Generalized false positive models

\section{Appendix S2. Additional details associated w/ model performance.}

Table S1. Scenarios considered when evaluating the Royle-Nichols and extended models. $\boldsymbol{\beta}$ describes abundance terms, $\boldsymbol{\alpha}$ describes detection terms. $\boldsymbol{X}_{\boldsymbol{I}}$ is a vector of simulated covariates that influences abundance, and for some scenarios, the likelihood of false positive observation.

\begin{tabular}{rccccc} 
Scenario & $\beta_{0}$ & $\beta_{1}$ & $\alpha_{0}$ & $\alpha_{1}$ & False Positive Model \\
\hline 1 & 0 & 1 & -1.73 & 1 & $\theta_{\mathrm{fp}}=0.10$ of $\theta_{\mathrm{fp}}+\theta_{\mathrm{tp}}$ \\
2 & 0 & 1 & -1.73 & 1 & $\theta_{\mathrm{fp}}=0.05$ of $\theta_{\mathrm{fp}}+\theta_{\mathrm{tp}}$ \\
3 & 0 & 1 & -1.73 & 1 & $\theta_{\mathrm{fp}}=0.01$ of $\theta_{\mathrm{fp}}+\theta_{\mathrm{tp}}$ \\
4 & -1.50 & 1 & -1.73 & 1 & $\theta_{\mathrm{fp}}=0.10$ of $\theta_{\mathrm{fp}}+\theta_{\mathrm{tp}}$ \\
5 & -1.50 & 1 & -1.73 & 1 & $\theta_{\mathrm{fp}}=0.05$ of $\theta_{\mathrm{fp}}+\theta_{\mathrm{tp}}$ \\
6 & -1.50 & 1 & -1.73 & 1 & $\theta_{\mathrm{fp}}=0.01$ of $\theta_{\mathrm{fp}}+\theta_{\mathrm{tp}}$ \\
7 & -1.50 & 1 & -1.73 & 1 & $\operatorname{logit}\left(\theta_{\mathrm{fp}, \mathrm{i}}\right)=-6+1 \mathrm{X}_{1, \mathrm{i}}$ \\
8 & -1.50 & 1 & -1.73 & 1 & $\operatorname{logit}\left(\theta_{\mathrm{fp}, \mathrm{i}}\right)=-6-1 \mathrm{X}_{1, \mathrm{i}}$
\end{tabular}


Generalized false positive models

Table S2. Mean error for parameters and relative bias of finite-sample population size for the standard and extended RN models across the scenarios considered.

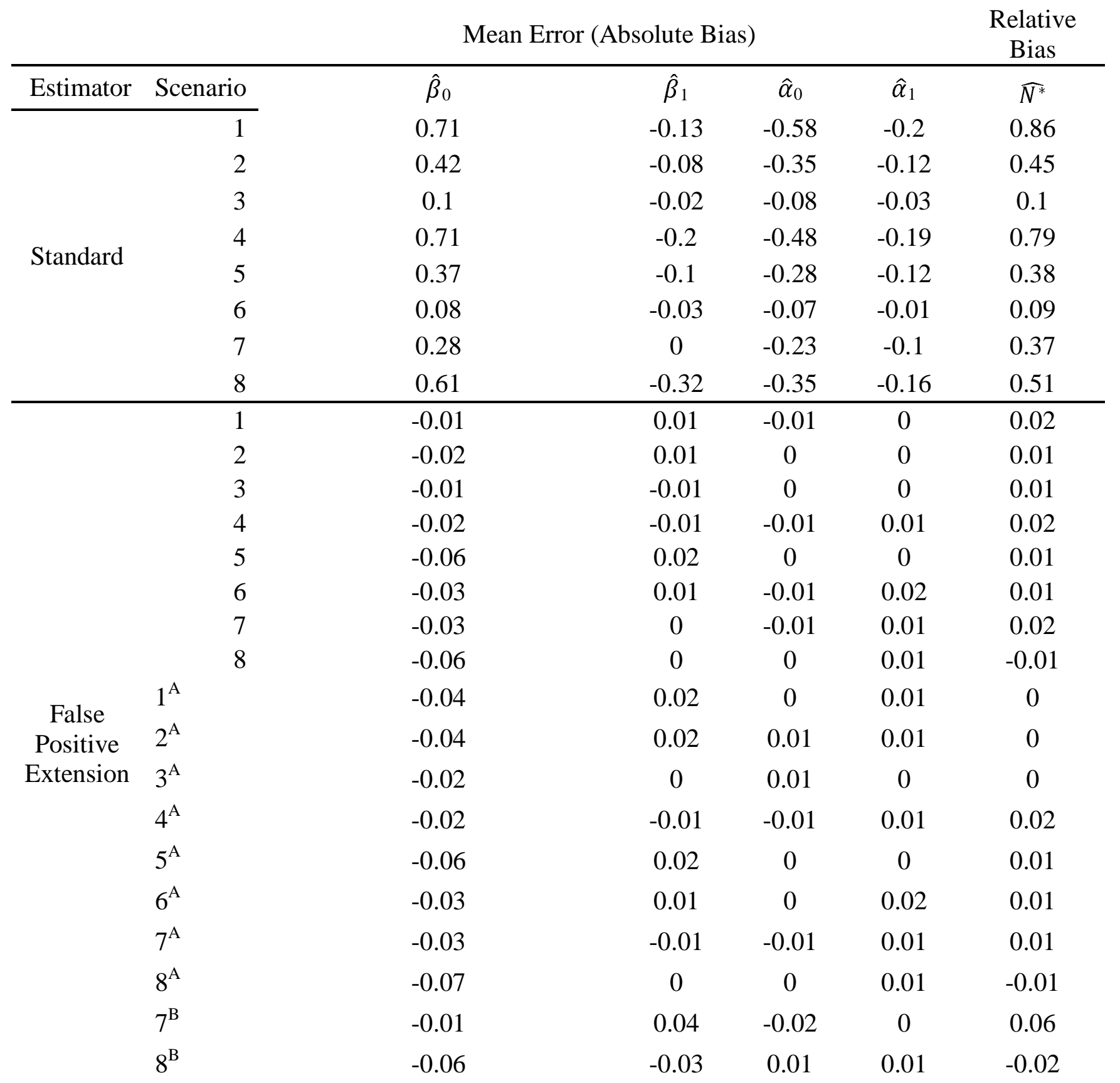

${ }^{\mathrm{A}}$ Fit using an informed prior for $\theta_{\mathrm{fp}}$ derived from estimates from an occupancy model following the observation confirmation protocol.

${ }^{\mathrm{B}}$ A slightly mis-specified model that assumes $\theta_{\mathrm{fp}}$ is constant. 
Generalized false positive models

Table S3. Frequentist coverage of 95\% CRI associated with parameters and finite-sample population size for the standard and extended RN models across the scenarios considered.

\begin{tabular}{|c|c|c|c|c|c|c|}
\hline & & & age & & & \\
\hline Estimator & Scenario & $\overline{\hat{\beta}_{0}}$ & $\hat{\beta}_{1}$ & $\hat{\alpha}_{0}$ & $\hat{\alpha}_{1}$ & $\widehat{N^{*}}$ \\
\hline & 1 & 0 & 0.403 & 0 & 0.216 & 0 \\
\hline & 2 & 0.093 & 0.756 & 0.176 & 0.556 & 0.083 \\
\hline & 3 & 0.84 & 0.913 & 0.87 & 0.89 & 0.866 \\
\hline & 4 & 0.066 & 0.556 & 0.086 & 0.523 & 0.013 \\
\hline Standard & 5 & 0.456 & 0.816 & 0.46 & 0.736 & 0.193 \\
\hline & 6 & 0.906 & 0.926 & 0.9 & 0.876 & 0.9 \\
\hline & 7 & 0.636 & 0.936 & 0.566 & 0.783 & 0.246 \\
\hline & 8 & 0.066 & 0.286 & 0.243 & 0.626 & 0.063 \\
\hline & 1 & 0.983 & 0.95 & 0.95 & 0.93 & 0.95 \\
\hline & 2 & 0.99 & 0.94 & 0.96 & 0.95 & 0.95 \\
\hline & 3 & 0.98 & 0.95 & 0.97 & 0.96 & 0.96 \\
\hline & 4 & 0.97 & 0.94 & 0.96 & 0.95 & 0.96 \\
\hline & 5 & 0.97 & 0.96 & 0.96 & 0.92 & 0.96 \\
\hline & 6 & 0.98 & 0.93 & 0.93 & 0.92 & 0.97 \\
\hline & 7 & 0.97 & 0.93 & 0.94 & 0.953 & 0.95 \\
\hline & 8 & 0.98 & 0.92 & 0.96 & 0.96 & 0.92 \\
\hline & $1^{\mathrm{A}}$ & 0.98 & 0.95 & 0.95 & 0.94 & 0.92 \\
\hline Positive & $2^{\mathrm{A}}$ & 0.99 & 0.95 & 0.96 & 0.95 & 0.96 \\
\hline Extension & $3^{A}$ & 0.99 & 0.94 & 0.96 & 0.96 & 0.96 \\
\hline & $4^{\mathrm{A}}$ & 0.98 & 0.94 & 0.97 & 0.95 & 0.96 \\
\hline & $5^{\mathrm{A}}$ & 0.98 & 0.96 & 0.96 & 0.93 & 0.94 \\
\hline & $6^{\mathrm{A}}$ & 0.98 & 0.92 & 0.92 & 0.92 & 0.98 \\
\hline & $7^{\mathrm{A}}$ & 0.973 & 0.94 & 0.94 & 0.96 & 0.95 \\
\hline & $8^{A}$ & 0.973 & 0.93 & 0.95 & 0.95 & 0.92 \\
\hline & $7^{\mathrm{B}}$ & 0.97 & 0.93 & 0.94 & 0.96 & 0.9 \\
\hline & $8^{\mathrm{B}}$ & 0.97 & 0.91 & 0.96 & 0.953 & 0.92 \\
\hline
\end{tabular}

${ }^{\mathrm{A}}$ Fit using an informed prior for $\theta_{\mathrm{fp}}$ derived from estimates from an occupancy model following the observation confirmation protocol.

${ }^{\mathrm{B}}$ A slightly mis-specified model that assumes $\theta_{\mathrm{fp}}$ is constant. 
Generalized false positive models

Table S4. Root mean squared error for parameters and relative bias of finite-sample population size for the standard and extended RN models across the scenarios considered.

\begin{tabular}{|c|c|c|c|c|c|c|}
\hline & & & & & & \\
\hline Estimator & Scenario & $\hat{\beta}_{0}$ & $\hat{\beta}_{1}$ & $\hat{\alpha}_{0}$ & $\hat{\alpha}_{1}$ & $\widehat{N^{*}}$ \\
\hline & 1 & 0.71 & 0.14 & 0.58 & 0.2 & 280 \\
\hline & 2 & 0.42 & 0.09 & 0.36 & 0.13 & 150 \\
\hline & 3 & 0.1 & 0.07 & 0.11 & 0.07 & 39 \\
\hline Stondord & 4 & 0.786 & 0.21 & 0.49 & 0.21 & 59 \\
\hline standara & 5 & 0.377 & 0.14 & 0.28 & 0.16 & 28 \\
\hline & 6 & 0.173 & 0.12 & 0.13 & 0.12 & 9 \\
\hline & 7 & 0.29 & 0.11 & 0.24 & 0.15 & 27 \\
\hline & 8 & 0.61 & 0.32 & 0.36 & 0.2 & 37 \\
\hline & 1 & 0.11 & 0.07 & 0.1 & 0.07 & 33 \\
\hline & 2 & 0.11 & 0.06 & 0.1 & 0.07 & 29 \\
\hline & 3 & 0.1 & 0.06 & 0.08 & 0.06 & 25 \\
\hline & 4 & 0.17 & 0.12 & 0.12 & 0.11 & 7.2 \\
\hline & 5 & 0.17 & 0.12 & 0.12 & 0.11 & 6.65 \\
\hline & 6 & 0.16 & 0.12 & 0.12 & 0.11 & 6 \\
\hline & 7 & 0.17 & 0.13 & 0.12 & 0.11 & 7.2 \\
\hline & 8 & 0.19 & 0.13 & 0.12 & 0.11 & 6.64 \\
\hline False & $1^{\mathrm{A}}$ & 0.12 & 0.07 & 0.1 & 0.07 & 32 \\
\hline Positive & $2^{\mathrm{A}}$ & 0.11 & 0.06 & 0.1 & 0.07 & 28 \\
\hline Extension & $3^{A}$ & 0.1 & 0.06 & 0.09 & 0.06 & 25 \\
\hline & $4^{\mathrm{A}}$ & 0.18 & 0.12 & 0.12 & 0.11 & 7.37 \\
\hline & $5^{\mathrm{A}}$ & 0.17 & 0.12 & 0.12 & 0.11 & 6.92 \\
\hline & $6^{\mathrm{A}}$ & 0.16 & 0.12 & 0.12 & 0.11 & 6.45 \\
\hline & $7^{\mathrm{A}}$ & 0.17 & 0.13 & 0.13 & 0.11 & 7.33 \\
\hline & $8^{\mathrm{A}}$ & 0.19 & 0.13 & 0.12 & 0.11 & 6.89 \\
\hline & $7^{\mathrm{B}}$ & 0.17 & 0.13 & 0.12 & 0.1 & 8.12 \\
\hline & $8^{\mathrm{B}}$ & 0.19 & 0.14 & 0.12 & 0.11 & 6.85 \\
\hline
\end{tabular}

${ }^{\mathrm{A}}$ Fit using an informed prior for $\theta_{\mathrm{fp}}$ derived from estimates from an occupancy model following the observation confirmation protocol.

${ }^{\mathrm{B}} \mathrm{A}$ slightly mis-specified model that assumes $\theta_{\mathrm{fp}}$ is constant. 
Generalized false positive models

Table S5. Scenarios considered when evaluating the arrival model of Roth et al. (2014) and extended models. $\boldsymbol{\beta}$ describes occupancy terms, $\boldsymbol{\alpha}$ describes detection terms. $\boldsymbol{X}_{\boldsymbol{I}}$ is a vector of simulated covariates that influences abundance, and for some scenarios, the likelihood of false positive observation. $\varphi$ denotes the simulated occasion of arrival. $\boldsymbol{X}_{\boldsymbol{I}}$ is a vector of simulated covariates that influences the probability of occupancy, and for some scenarios, the likelihood of false positive observation.

\begin{tabular}{llllllll} 
Scenario & $\beta_{0}$ & $\beta_{1}$ & $\alpha_{0}$ & $\alpha_{1}$ & $\varphi$ & $\begin{array}{l}\text { Start of False } \\
\text { Positives }\end{array}$ & False Positive Model \\
\hline 1 & 0 & 0.5 & -2 & 0.5 & 6 & $>0$ & $\theta_{\mathrm{fp}}=0.10$ of $\theta_{\mathrm{fp}}+\theta_{\mathrm{tp}}$ \\
2 & 0 & 0.5 & -2 & 0.5 & 6 & $>0$ & $\theta_{\mathrm{fp}}=0.05$ of $\theta_{\mathrm{fp}}+\theta_{\mathrm{tp}}$ \\
3 & 0 & 0.5 & -2 & 0.5 & 6 & $>0$ & $\theta_{\mathrm{fp}}=0.01$ of $\theta_{\mathrm{fp}}+\theta_{\mathrm{tp}}$ \\
4 & 0 & 0.5 & -2 & 0.5 & 6 & 5 & $\operatorname{logit}\left(\theta_{\mathrm{fp}, \mathrm{i}}\right)=-6+1 \mathrm{X}_{1, \mathrm{i}}$ \\
5 & 0 & 0.5 & -2 & 0.5 & 6 & 5 & $\operatorname{logit}\left(\theta_{\mathrm{fp}, \mathrm{i}}\right)=-6-1 \mathrm{X}_{1, \mathrm{i}}$ \\
6 & 0 & 0.5 & -2 & 0.5 & 6 & 3 & $\operatorname{logit}\left(\theta_{\mathrm{fp}, \mathrm{i}}\right)=-6+1 \mathrm{X}_{1, \mathrm{i}}$ \\
7 & 0 & 0.5 & -2 & 0.5 & 6 & 3 & $\operatorname{logit}\left(\theta_{\mathrm{fp}, \mathrm{i}}\right)=-6-1 \mathrm{X}_{1, \mathrm{i}}$ \\
8 & 0 & 0.5 & -1 & 0.5 & 6 & 5 & $\operatorname{logit}\left(\theta_{\mathrm{fp}, \mathrm{i}}\right)=-6+1 \mathrm{X}_{1, \mathrm{i}}$ \\
9 & 0 & 0.5 & -1 & 0.5 & 6 & 5 & $\operatorname{logit}\left(\theta_{\mathrm{fp}, \mathrm{i}}\right)=-6-1 \mathrm{X}_{1, \mathrm{i}}$
\end{tabular}


Generalized false positive models

Table S6. Estimator error and relative bias associated with parameters using the standard and extended arrival occupancy models across the scenarios in table S5.

Mean Error (Absolute Bias)

Relative Bias

\begin{tabular}{|c|c|c|c|c|c|c|c|}
\hline Estimator & Scenario & $\hat{\beta}_{0}$ & $\hat{\beta}_{1}$ & $\hat{\alpha}_{0}$ & $\hat{\alpha}_{1}$ & $\widehat{P A O}$ & $\hat{\varphi}$ \\
\hline \multirow{9}{*}{ Standard } & 1 & 0.36 & 0.03 & -0.22 & -0.04 & 0.16 & -0.42 \\
\hline & 2 & 0.18 & 0.02 & -0.13 & -0.02 & 0.09 & -0.22 \\
\hline & 3 & 0.07 & 0.03 & -0.04 & -0.01 & 0.03 & -0.03 \\
\hline & 4 & 0.19 & 0.17 & -0.01 & -0.02 & 0.08 & -0.01 \\
\hline & 5 & 0.24 & -0.21 & -0.04 & -0.02 & 0.11 & -0.01 \\
\hline & 6 & 0.18 & 0.17 & -0.04 & -0.03 & 0.08 & -0.1 \\
\hline & 7 & 0.28 & -0.24 & -0.1 & -0.03 & 0.13 & -0.1 \\
\hline & 8 & 0.11 & 0.13 & -0.04 & -0.01 & 0.05 & 0 \\
\hline & 9 & 0.17 & -0.15 & -0.07 & -0.01 & 0.08 & 0.01 \\
\hline \multirow{23}{*}{$\begin{array}{l}\text { False } \\
\text { Positive } \\
\text { Extension }\end{array}$} & 1 & 0.03 & 0.07 & -0.01 & 0 & 0.01 & 0.02 \\
\hline & 2 & 0 & 0.05 & -0.01 & 0 & 0.01 & 0.02 \\
\hline & 3 & 0.01 & 0.03 & -0.02 & -0.01 & 0 & 0.02 \\
\hline & 4 & 0.05 & 0.09 & -0.01 & 0 & 0.02 & 0.01 \\
\hline & 5 & 0.02 & -0.01 & 0 & 0 & 0.01 & 0.02 \\
\hline & 6 & 0 & 0.06 & 0 & 0 & 0 & 0.02 \\
\hline & 7 & 0.02 & 0 & -0.11 & 0 & 0.01 & 0.02 \\
\hline & 8 & 0 & 0.07 & 0 & 0 & 0.01 & 0.02 \\
\hline & 9 & 0.01 & 0.02 & -0.01 & 0.01 & 0 & 0.02 \\
\hline & $1^{\mathrm{A}}$ & 0.03 & 0.08 & -0.01 & 0 & 0.01 & 0.02 \\
\hline & $2^{\mathrm{A}}$ & -0.06 & 0.06 & -0.01 & 0.01 & -0.02 & 0.02 \\
\hline & $3^{A}$ & -0.1 & 0.05 & -0.01 & 0 & -0.04 & 0.03 \\
\hline & $4^{\mathrm{A}}$ & 0.06 & 0.11 & -0.01 & 0 & 0.03 & 0.01 \\
\hline & $5^{\mathrm{A}}$ & 0.05 & 0.04 & 0.01 & 0.01 & 0.04 & 0.03 \\
\hline & $6^{\mathrm{A}}$ & -0.02 & 0.06 & -0.01 & 0 & 0.03 & 0.02 \\
\hline & $7^{\mathrm{A}}$ & 0.06 & 0.05 & -0.01 & 0.01 & 0.03 & 0.03 \\
\hline & $8^{\mathrm{A}}$ & 0.04 & 0.04 & -0.01 & 0 & 0.02 & 0.02 \\
\hline & $9^{\mathrm{A}}$ & 0.03 & 0.02 & 0.02 & 0 & -0.05 & 0.02 \\
\hline & $4^{\mathrm{B}}$ & 0.06 & 0.13 & 0 & 0 & 0.03 & 0.01 \\
\hline & $5^{\mathrm{B}}$ & 0.06 & -0.1 & -0.01 & 0 & 0.03 & 0.02 \\
\hline & $6^{\mathrm{B}}$ & 0.01 & 0.13 & 0.01 & 0 & 0 & 0.01 \\
\hline & $7^{\mathrm{B}}$ & 0.05 & -0.11 & -0.02 & 0 & 0.02 & 0.02 \\
\hline & $8^{\mathrm{B}}$ & 0.02 & 0.1 & 0 & 0 & 0.01 & 0.01 \\
\hline
\end{tabular}


Generalized false positive models

\begin{tabular}{clcccccc} 
& $9^{\mathrm{B}}$ & 0.03 & -0.03 & -0.01 & 0.01 & 0.01 & 0.02 \\
\cline { 2 - 8 } & 1 & 0.04 & 0.07 & -0.01 & 0 & 0.02 & 0.02 \\
Standard & 2 & 0.02 & 0.04 & -0.01 & 0 & 0.02 & 0.02 \\
Estimator, & 3 & 0.04 & 0.04 & -0.02 & 0.01 & 0.02 & 0.02 \\
fit only & 4 & 0.05 & 0.05 & -0.02 & 0.01 & 0.02 & 0.01 \\
using & 5 & 0.02 & 0.05 & 0.00 & 0.00 & 0.01 & 0.02 \\
simulated & 6 & 0.03 & 0.04 & 0.00 & 0.00 & 0.01 & 0.02 \\
true & 7 & 0.03 & 0.03 & -0.02 & 0.00 & 0.01 & 0.02 \\
positives & 8 & 0.00 & 0.04 & 0.00 & 0.00 & 0.00 & 0.02 \\
& 9 & 0.01 & 0.04 & -0.01 & 0.01 & 0.00 & 0.02
\end{tabular}

${ }^{A}$ Fit using an informed prior for $\theta_{\mathrm{fp}}$ derived from estimates from an occupancy model following the observation confirmation protocol.

${ }^{\mathrm{B}} \mathrm{A}$ slightly mispecified model that assumes $\theta_{\mathrm{fp}}$ is constant. 
Generalized false positive models

Table S7. Frequentist coverage of $95 \%$ CRI associated with parameters using the standard and extended arrival occupancy models across the scenarios in table S5.

Coverage

\begin{tabular}{|c|c|c|c|c|c|c|c|}
\hline Estimator & Scenario & $\hat{\beta}_{0}$ & $\hat{\beta}_{1}$ & $\hat{\alpha}_{0}$ & $\hat{\alpha}_{1}$ & $\widehat{P A O}$ & $\hat{\varphi}$ \\
\hline \multirow{9}{*}{ Standard } & 1 & 0.54 & 0.94 & 0.41 & 0.9 & 0.25 & 0.1 \\
\hline & 2 & 0.84 & 0.94 & 0.71 & 0.93 & 0.64 & 0.35 \\
\hline & 3 & 0.94 & 0.93 & 0.94 & 0.95 & 0.93 & 0.82 \\
\hline & 4 & 0.85 & 0.9 & 0.92 & 0.92 & 0.65 & 0.91 \\
\hline & 5 & 0.81 & 0.76 & 0.91 & 0.94 & 0.53 & 0.93 \\
\hline & 6 & 0.84 & 0.87 & 0.94 & 0.93 & 0.71 & 0.5 \\
\hline & 7 & 0.67 & 0.74 & 0.85 & 0.97 & 0.4 & 0.53 \\
\hline & 8 & 0.89 & 0.86 & 0.87 & 0.91 & 0.26 & 0.92 \\
\hline & 9 & 0.82 & 0.84 & 0.78 & 0.9 & 0.08 & 0.95 \\
\hline \multirow{24}{*}{$\begin{array}{c}\text { False } \\
\text { Positive } \\
\text { Extension }\end{array}$} & 1 & 0.96 & 0.93 & 0.95 & 0.96 & 0.97 & 0.93 \\
\hline & 2 & 0.96 & 0.93 & 0.96 & 0.95 & 0.95 & 0.95 \\
\hline & 3 & 0.95 & 0.92 & 0.97 & 0.95 & 0.96 & 0.94 \\
\hline & 4 & 0.95 & 0.94 & 0.92 & 0.96 & 0.94 & 0.93 \\
\hline & 5 & 0.96 & 0.95 & 0.93 & 0.95 & 0.94 & 0.93 \\
\hline & 6 & 0.95 & 0.93 & 0.95 & 0.94 & 0.97 & 0.96 \\
\hline & 7 & 0.94 & 0.94 & 0.95 & 0.97 & 0.96 & 0.94 \\
\hline & 8 & 0.95 & 0.93 & 0.95 & 0.95 & 0.97 & 0.90 \\
\hline & 9 & 0.96 & 0.95 & 0.93 & 0.96 & 0.97 & 0.94 \\
\hline & $1^{\mathrm{A}}$ & 0.97 & 0.93 & 0.97 & 0.95 & 0.97 & 0.94 \\
\hline & $2^{\mathrm{A}}$ & 0.95 & 0.93 & 0.96 & 0.94 & 0.94 & 0.94 \\
\hline & $3^{\mathrm{A}}$ & 0.91 & 0.93 & 0.97 & 0.96 & 0.91 & 0.92 \\
\hline & $4^{\mathrm{A}}$ & 0.94 & 0.94 & 0.93 & 0.95 & 0.96 & 0.94 \\
\hline & $5^{\mathrm{A}}$ & 0.92 & 0.94 & 0.95 & 0.94 & 0.96 & 0.92 \\
\hline & $6^{\mathrm{A}}$ & 0.93 & 0.95 & 0.96 & 0.93 & 0.95 & 0.95 \\
\hline & $7^{\mathrm{A}}$ & 0.94 & 0.94 & 0.95 & 0.94 & 0.96 & 0.94 \\
\hline & $8^{\mathrm{A}}$ & 0.92 & 0.94 & 0.94 & 0.96 & 0.96 & 0.90 \\
\hline & $9^{\mathrm{A}}$ & 0.94 & 0.93 & 0.94 & 0.95 & 0.96 & 0.93 \\
\hline & $4^{\mathrm{B}}$ & 0.94 & 0.9 & 0.92 & 0.95 & 0.93 & 0.92 \\
\hline & $5^{\mathrm{B}}$ & 0.94 & 0.9 & 0.93 & 0.95 & 0.92 & 0.93 \\
\hline & $6^{\mathrm{B}}$ & 0.95 & 0.92 & 0.94 & 0.94 & 0.98 & 0.95 \\
\hline & $7^{\mathrm{B}}$ & 0.93 & 0.9 & 0.95 & 0.97 & 0.93 & 0.93 \\
\hline & $8^{\mathrm{B}}$ & 0.93 & 0.91 & 0.95 & 0.95 & 0.93 & 0.91 \\
\hline & $9^{\mathrm{B}}$ & 0.96 & 0.94 & 0.93 & 0.95 & 0.97 & 0.92 \\
\hline
\end{tabular}

${ }^{\mathrm{A}}$ Fit using an informed prior for $\theta$ fp derived from estimates from an occupancy model following the observation confirmation protocol.

${ }^{\mathrm{B}}$ A slightly mis-specified model that assumes $\theta \mathrm{fp}$ is constant. 
Generalized false positive models

Table S8. Root mean squared error associated with parameters using the standard and extended arrival occupancy models across the scenarios in table S5.

RMSE

\begin{tabular}{|c|c|c|c|c|c|c|c|}
\hline Estimator & Scenario & $\hat{\beta}_{0}$ & $\hat{\beta}_{1}$ & $\hat{\alpha}_{0}$ & $\hat{\alpha}_{1}$ & $\widehat{P A O}$ & $\hat{\varphi}$ \\
\hline \multirow{9}{*}{ Standard } & 1 & 0.36 & 0.18 & 0.224 & 0.08 & 0.08 & 25.2 \\
\hline & 2 & 0.23 & 0.17 & 0.15 & 0.08 & 0.05 & 13.6 \\
\hline & 3 & 0.17 & 0.16 & 0.1 & 0.08 & 0.03 & 4 \\
\hline & 4 & 0.23 & 0.21 & 0.09 & 0.08 & 0.04 & 2.3 \\
\hline & 5 & 0.25 & 0.24 & 0.1 & 0.07 & 0.06 & 2.4 \\
\hline & 6 & 0.22 & 0.22 & 0.09 & 0.08 & 0.04 & 6.4 \\
\hline & 7 & 0.3 & 0.26 & 0.12 & 0.08 & 0.07 & 6.5 \\
\hline & 8 & 0.15 & 0.18 & 0.06 & 0.06 & 0.03 & 1.5 \\
\hline & 9 & 0.19 & 0.18 & 0.09 & 0.06 & 0.04 & 1.4 \\
\hline \multirow{24}{*}{$\begin{array}{c}\text { False } \\
\text { Positive } \\
\text { Extension }\end{array}$} & 1 & 0.16 & 0.19 & 0.09 & 0.08 & 0.03 & 2.76 \\
\hline & 2 & 0.16 & 0.17 & 0.09 & 0.08 & 0.03 & 2.45 \\
\hline & 3 & 0.16 & 0.17 & 0.08 & 0.08 & 0.02 & 2.4 \\
\hline & 4 & 0.18 & 0.18 & 0.1 & 0.08 & 0.03 & 2.45 \\
\hline & 5 & 0.16 & 0.17 & 0.1 & 0.07 & 0.03 & 2.7 \\
\hline & 6 & 0.17 & 0.18 & 0.09 & 0.08 & 0.02 & 2.3 \\
\hline & 7 & 0.17 & 0.17 & 0.09 & 0.08 & 0.03 & 2.6 \\
\hline & 8 & 0.13 & 0.15 & 0.06 & 0.06 & 0.01 & 1.54 \\
\hline & 9 & 0.12 & 0.13 & 0.06 & 0.06 & 0.01 & 1.66 \\
\hline & $1^{\mathrm{A}}$ & 0.17 & 0.2 & 0.09 & 0.08 & 0.03 & 2.84 \\
\hline & $2^{\mathrm{A}}$ & 0.18 & 0.18 & 0.09 & 0.08 & 0.03 & 2.67 \\
\hline & $3^{\mathrm{A}}$ & 0.19 & 0.17 & 0.09 & 0.08 & 0.03 & 2.73 \\
\hline & $4^{\mathrm{A}}$ & 0.19 & 0.19 & 0.10 & 0.08 & 0.03 & 2.45 \\
\hline & $5^{\mathrm{A}}$ & 0.18 & 0.18 & 0.10 & 0.07 & 0.03 & 2.74 \\
\hline & $6^{\mathrm{A}}$ & 0.19 & 0.18 & 0.09 & 0.08 & 0.02 & 2.4 \\
\hline & $7^{\mathrm{A}}$ & 0.19 & 0.18 & 0.09 & 0.08 & 0.03 & 2.7 \\
\hline & $8^{\mathrm{A}}$ & 0.14 & 0.15 & 0.07 & 0.06 & 0.01 & 1.7 \\
\hline & $9^{\mathrm{A}}$ & 0.14 & 0.14 & 0.06 & 0.06 & 0.01 & 1.8 \\
\hline & $4^{\mathrm{B}}$ & 0.18 & 0.2 & 0.1 & 0.07 & 0.03 & 2.4 \\
\hline & $5^{\mathrm{B}}$ & 0.16 & 0.19 & 0.1 & 0.07 & 0.03 & 2.7 \\
\hline & $6^{\mathrm{B}}$ & 0.18 & 0.2 & 0.09 & 0.08 & 0.02 & 2.3 \\
\hline & $7^{\mathrm{B}}$ & 0.17 & 0.19 & 0.09 & 0.08 & 0.03 & 2.6 \\
\hline & $8^{\mathrm{B}}$ & 0.13 & 0.16 & 0.06 & 0.06 & 0.01 & 1.5 \\
\hline & $9^{\mathrm{B}}$ & 0.12 & 0.14 & 0.06 & 0.06 & 0.01 & 1.7 \\
\hline
\end{tabular}

${ }^{\mathrm{A}}$ Fit using an informed prior for $\theta_{\mathrm{fp}}$ derived from estimates from an occupancy model following the observation confirmation protocol.

${ }^{\mathrm{B}}$ A slightly mispecified model that assumes $\theta_{\mathrm{fp}}$ is constant. 
bioRxiv preprint doi: https://doi.org/10.1101/422527; this version posted September 17,2019 . The copyright holder for this preprint (which was not certified by peer review) is the author/funder, who has granted bioRxiv a license to display the preprint in perpetuity. It is made available under aCC-BY-NC-ND 4.0 International license.

Generalized false positive models

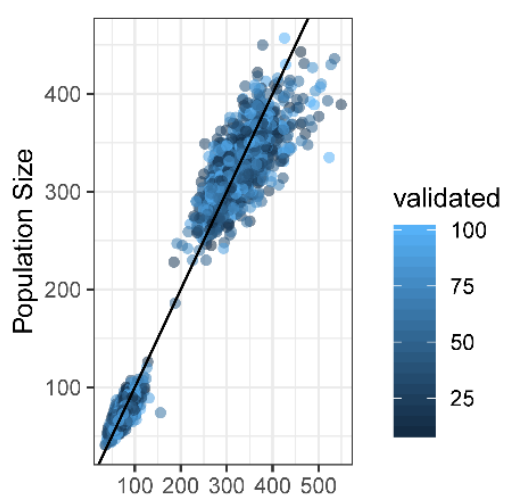

Estimated Population Size
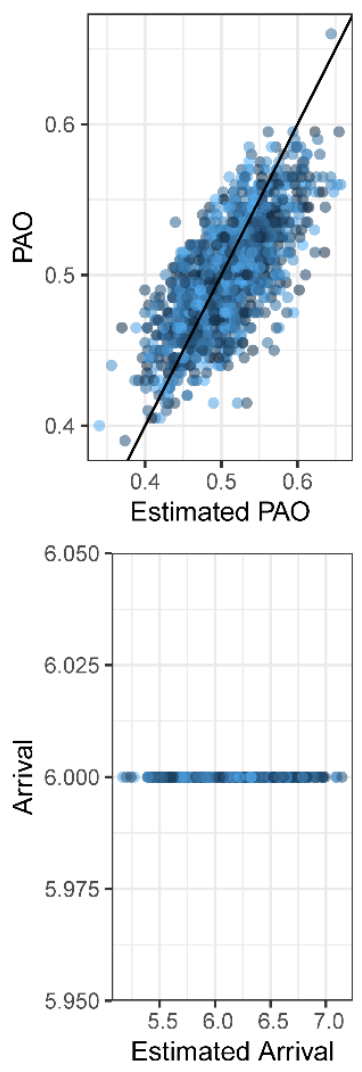

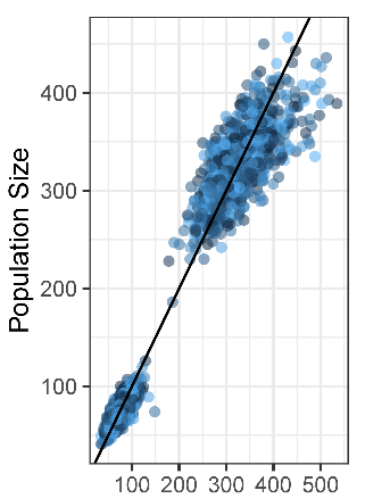

Estimated Population Size, Informed Prior

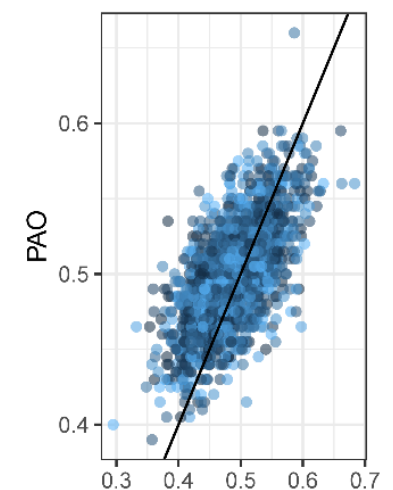

Estimated PAO, Informed Prior

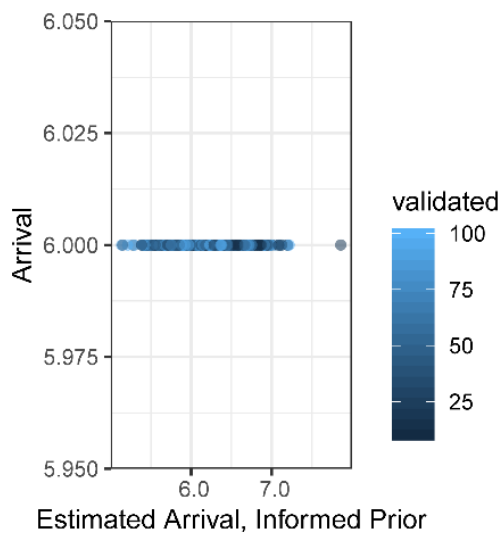

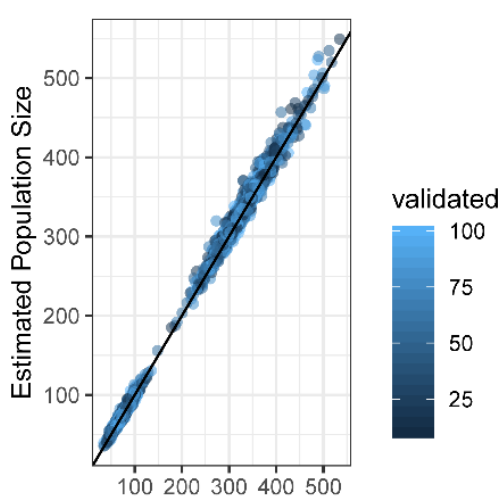

Estimated Population Size, Informed Prior

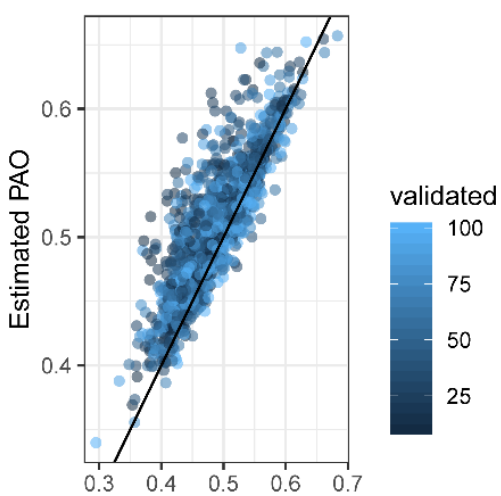

Estimated PAO, Informed Prior

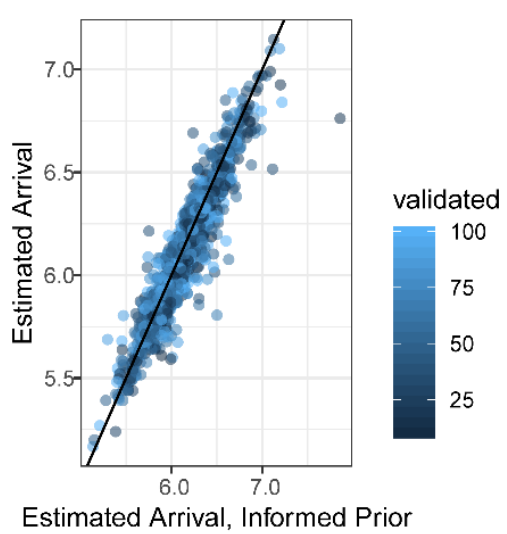

Figure S1. Correlation between true values, estimates made when incorporating confirmed observations, and estimates made when using an informed prior rather than incorporating confirmed observations in the likelihood. 
Generalized false positive models
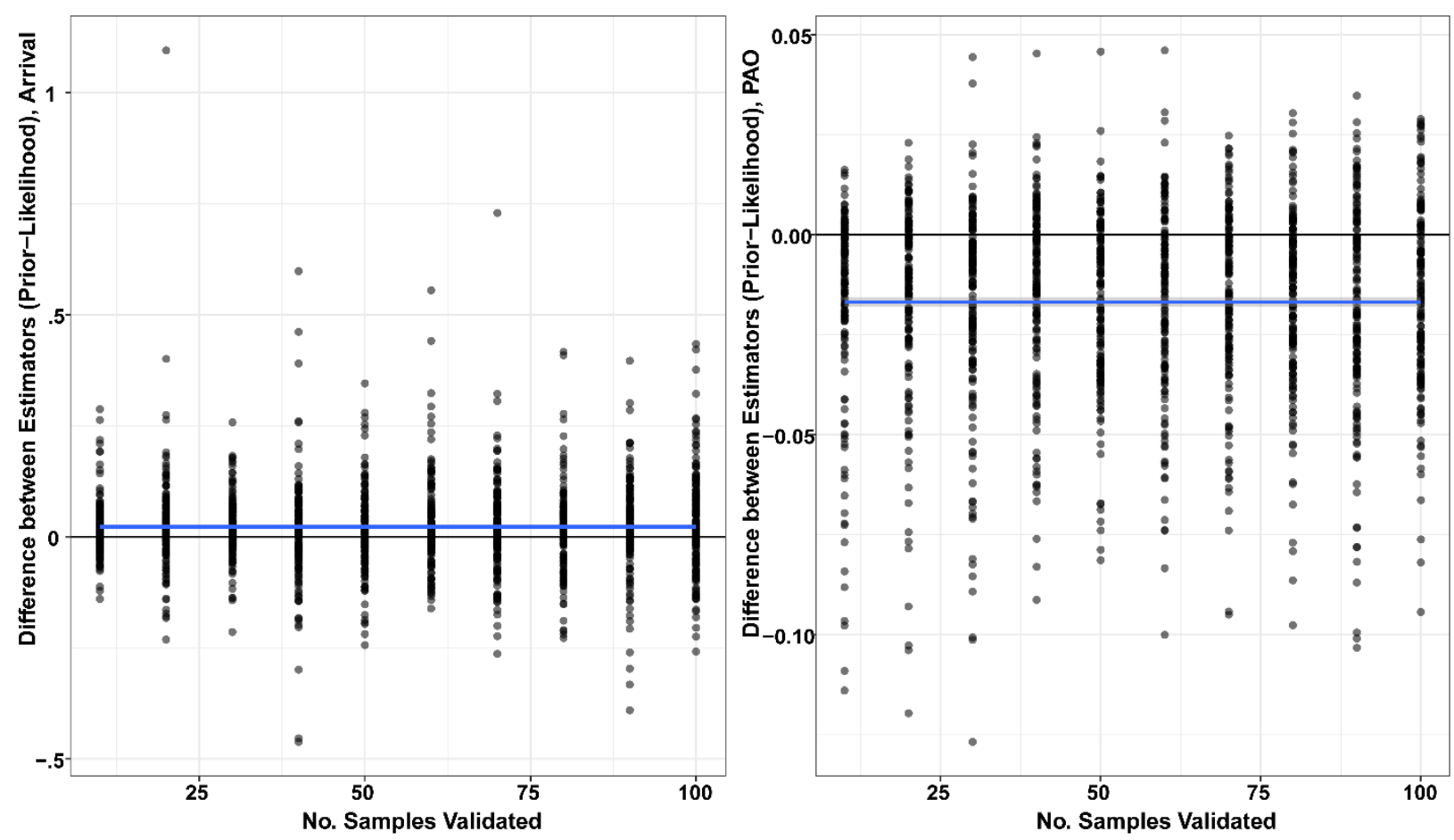

Figure S2. Noise in the correlations between estimates of PAO and arrival generated when including confirmed data in the likelihood vs. using an informed prior is not related to the size of the confirmation sample. 
Generalized false positive models

Appendix S3. Details associated w/ case study.

The data used here were trail camera images classified via crowdsourcing from 91,276 24-h periods at 944 distinct locations across the state in 2017 between Julian days 150 and 320 (Figure S1). We defined sampling occasions as 24-hr periods, and reviewed all reported images reported as gray fox classifications ( $\mathrm{n}=247$ images) from 179 occasions at 127 locations; 90 other occasions at 55 distinct locations included putative but unconfirmed gray fox detections (Figure S1). Covariates for expected abundance are noted in Table S1 and were extracted from a circular buffer of 1 or $5 \mathrm{~km}$ radius surrounding the camera locations. Spatial smoothing was implemented via a 2-dimensional cubic spline (Guélat and Kéry 2018) across latitude and longitude with 20 knots placed across the state. The detection probability of an individual animal at different sites was modeled as varying in relation to whether the camera was placed on a maintained trail or not and as a quadratic function of the distance between the camera and the location the camera was targeting (as reported by volunteers), and false positive error probability was modeled as a logistic function of the proportion of cropland within a circular buffer with 5 $\mathrm{km}$ radius to account for what we expected to be increased prevalence of species confused with gray foxes (red fox, coyote) relative to foxes themselves. The prediction grain (a $2 \times 2 \mathrm{~km}$ lattice) was chosen to approximate gray fox home range sizes in Wisconsin, which are believed to be slightly larger than the home ranges reported slightly further south (e.g., Haroldson and Fritzell 1984, Duell et al. 2017).

In Clare et al. (2019), we noted that perhaps the most useful predictor for classification error across a range of species was the degree of unanimity in the crowdsourced classifications (e.g., $100 \%$ of votes for gray fox $=$ more likely to be gray fox than $50 \%$ votes). We do not use this term here to avoid the inelegance of having to define/impute values associated with 
Generalized false positive models

crowdsourced agreement within sampling occasions in which the gray fox was not detected, although following the logic that greater confidence leads to lower probability of a false positive, imputing these values as 1.0 (i.e., 100\% agreement) seems like a reasonable hack. Similar inelegancies associated with agreement arise for sampling occasions with multiple images; one way to model this that might be reasonable might be to define a covariate (or the false positive probability) as $1-\prod_{p=1}^{n p i c t u r e s}\left(1-\right.$ agreement $\left._{p}\right)$. For example, in a case with 2 pictures in with 50 and $40 \%$ agreement, the value of the operator $=0.7$, in a case with 1 picture with $90 \%$ agreement, the value $=0.1$, which seems to correctly imply a false positive is more likely in the first case because there are more images with less confidence. Of course, in situations with 0 pictures, the operator breaks down, and the value might need to be fixed at one.

Of the reviewed images, we confirmed $67 \%$ as correct classifications, with the rest either misclassifications of coyote (Canis latrans) or red fox (Vulpes vulpes). Once aggregated within sampling occasions, $60 \%$ (108) of the occasions consisted exclusively of true positives, and $40 \%$ (71) included exclusively false positives; no confirmed sampling intervals included a mixture of true and false positive observations. This might suggest some potential lack of independence between true and false positive outcomes, but we ignore that here because volunteers view images on the crowdsourcing platform at random, which makes it difficult to imagine that misperception has any serial structure or exhibits any other form of dependence. We note that both true and false positives were reported at 6 locations. Estimates are summarized in tables S2 and S3.

\section{References}


bioRxiv preprint doi: https://doi.org/10.1101/422527; this version posted September 17, 2019. The copyright holder for this preprint (which was not certified by peer review) is the author/funder, who has granted bioRxiv a license to display the preprint in perpetuity. It is made available under aCC-BY-NC-ND 4.0 International license.

Generalized false positive models

Deuel, N.R., Conner, L.M., Miller, K.V., Chamberlain, M.J., Cherry, M.J. and Tannenbaum, L.V. 2017. Gray fox home range, spatial overlap, mated pair interactions and extra-territorial forays in southwestern Georgia, USA. Wildlife Biology: https://doi.org/10.2981/wlb.00326.

Guélat, J. and Kéry, M. 2018. Effects of spatial autocorrelation and imperfect detection on species distribution models. Methods in Ecology and Evolution 9:1614-1625.

Haroldson, K.J. and Fritzell, E.K. 1984. Home ranges, activity, and habitat use by gray foxes in an oak-hickory forest. The Journal of Wildlife Management 48:222-227.

\section{Gray Fox Observations 2017}

A)

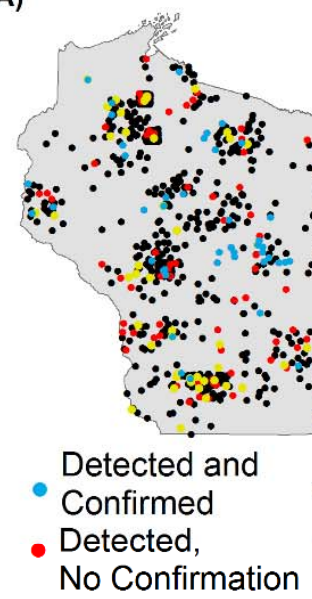

\section{B)}

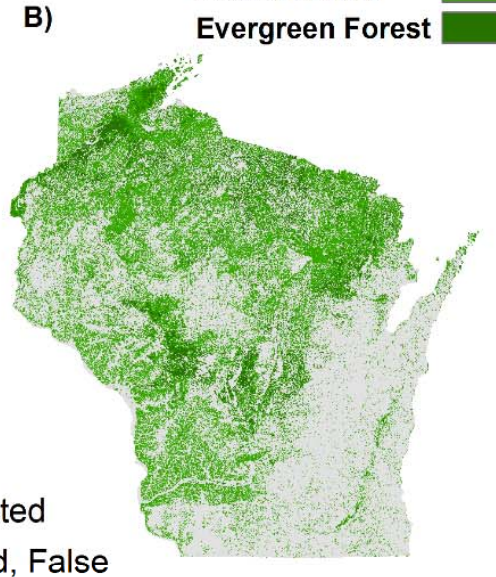

Confirmed, False

Positives Only
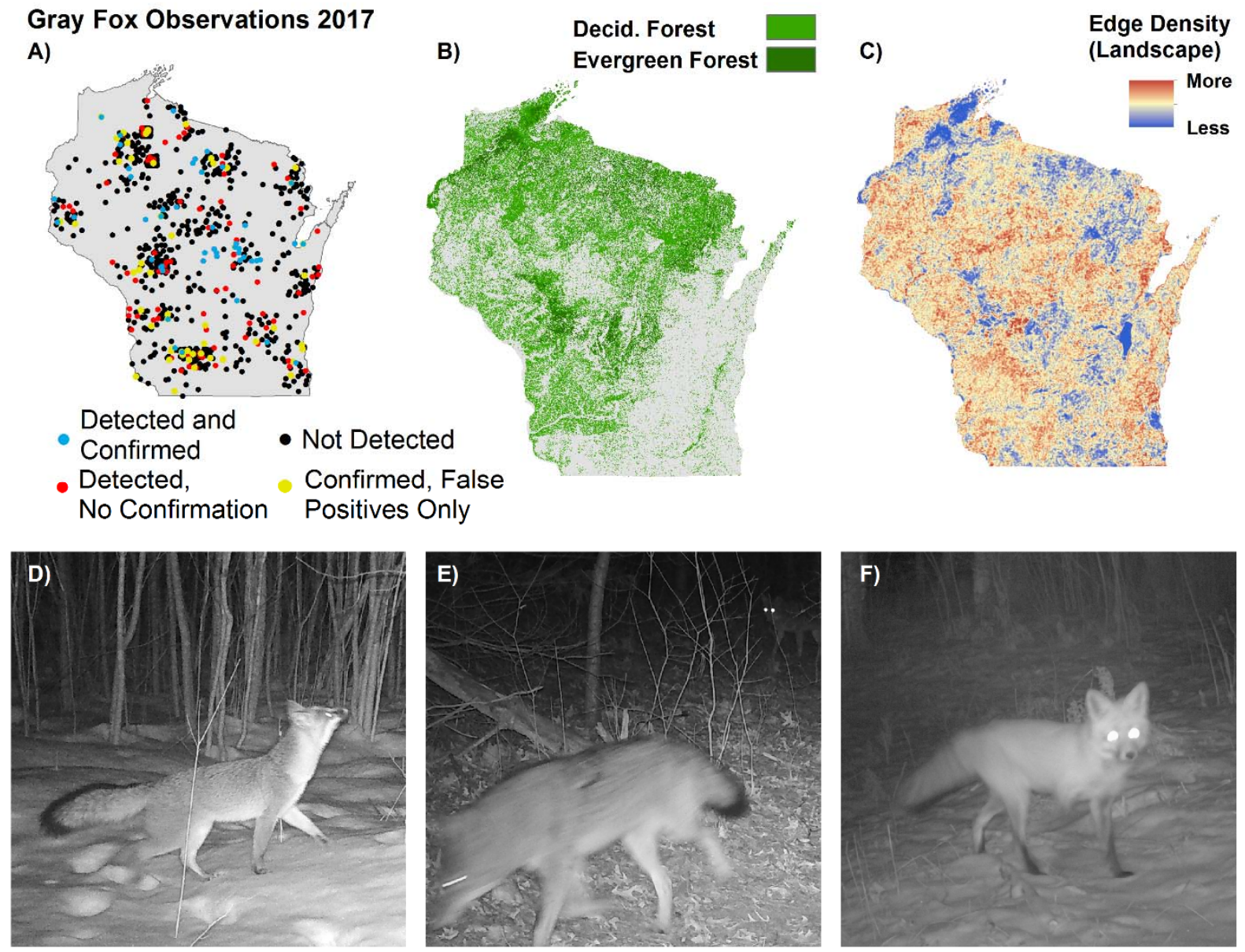
Generalized false positive models

Figure S1. Location of sampling locations, confirmed observations, and unconfirmed detections used in the case study (A); covariates associated with model fitting (B and C). Reported gray fox (D) observations were commonly truly either coyote (E) or red fox (F).

Table S1.

\begin{tabular}{|c|c|c|c|}
\hline Name & Description & Source & $\begin{array}{l}\text { Real } \\
\text { Parameter }\end{array}$ \\
\hline Evergreen & $\begin{array}{l}\% \text { Evergreen Forest in } 1 \mathrm{~km} \text { radius buffer surrounding camera } \\
\text { location }\end{array}$ & NLCD $2011^{\mathrm{A}}$ & $\lambda$ \\
\hline Snowdepth & $\begin{array}{l}\text { Mean Snowdepth in } 1 \mathrm{~km} \text { radius buffer surrounding camera location } \\
\text { between } 2010 \text { and } 2017\end{array}$ & SNODAS $^{\mathrm{B}}$ & $\lambda$ \\
\hline $\begin{array}{l}\text { Edge } \\
\text { Density }\end{array}$ & $\begin{array}{l}\text { (Landscape) edge density within } 1 \mathrm{~km} \text { radius buffer surrounding } \\
\text { camera location }\end{array}$ & NLCD 2011 & $\lambda$ \\
\hline Cropland & $\%$ Cropland in $1 \mathrm{~km}$ radius buffer surrounding camera location & NLCD 2011 & $\lambda, s_{0}$ \\
\hline Grassland & $\%$ Grassland in $1 \mathrm{~km}$ radius buffer surrounding camera location & NLCD 2011 & $\lambda$ \\
\hline Deciduous & $\begin{array}{l}\% \text { Deciduous forest in } 1 \mathrm{~km} \text { radius buffer surrounding camera } \\
\text { location }\end{array}$ & NLCD 2011 & $\lambda$ \\
\hline Trail & $\begin{array}{l}\text { Binary; camera placed on maintained trail (vs. game trail or non- } \\
\text { trail); }\end{array}$ & & $\mathrm{r}$ \\
\hline Distance & Distance between camera and target location $(\mathrm{m})$; modeled as quadratic & & $\mathrm{r}$ \\
\hline \multicolumn{4}{|c|}{${ }^{\text {A} H o m e r, ~ C ., ~ D e w i t z, ~ J ., ~ Y a n g, ~ L ., ~ J i n, ~ S ., ~ D a n i e l s o n, ~ P ., ~ X i a n, ~ G ., ~ C o u l s t o n, ~ J ., ~ H e r o l d, ~ N ., ~}$} \\
\hline \multicolumn{4}{|c|}{$\begin{array}{l}\text { Wickham, J. and Megown, K., 2015. Completion of the } 2011 \text { National Land Cover Database for } \\
\text { the conterminous United States-representing a decade of land cover change } \\
\text { information. Photogrammetric Engineering \& Remote Sensing 81:345-354. }\end{array}$} \\
\hline \multicolumn{4}{|c|}{${ }^{\mathrm{B}}$ Barrett, A. 2003. National Operational Hydrologic Remote Sensing Center SNOw Data } \\
\hline \multicolumn{4}{|c|}{$\begin{array}{l}\text { Assimilation System (SNODAS) Products at NSIDC. NSIDC Special Report 11. Boulder, CO, } \\
\text { USA: National Snow and Ice Data Center. Digital media. }\end{array}$} \\
\hline
\end{tabular}


Generalized false positive models

Table S2. Parameter estimates, variable inclusion probabilities $(w)$, and $95 \%$ credible intervals (LCI, UCI) from a RN model for gray fox relative abundance ignoring false positive error.

\begin{tabular}{|c|c|c|c|}
\hline Parameter & Estimate & LCI & UCI \\
\hline Intercept, $\lambda$ & -1.069 & -2.573 & 1.107 \\
\hline Evergreen & -0.381 & -2.988 & 2.148 \\
\hline Snowdepth & 0.003 & -6.171 & 6.197 \\
\hline Edge Density & -0.031 & -6.218 & 6.088 \\
\hline Cropland & -0.009 & -6.125 & 6.166 \\
\hline Grassland & 0.011 & -6.174 & 6.117 \\
\hline Deciduous & -0.011 & -6.051 & 6.054 \\
\hline Evergreen, $\mathrm{w}^{\mathrm{A}}$ & 0.889 & 0 & 1 \\
\hline Snowdepth, $\mathrm{w}^{\mathrm{A}}$ & 0.034 & 0 & 1 \\
\hline Edge Density, $\mathrm{w}^{\mathrm{A}}$ & 0.028 & 0 & 1 \\
\hline Cropland, $\mathrm{w}^{\mathrm{A}}$ & 0.042 & 0 & 1 \\
\hline Grassland, $\mathrm{w}^{\mathrm{A}}$ & 0.068 & 0 & 1 \\
\hline Deciduous, $\mathrm{w}^{\mathrm{A}}$ & 0.048 & 0 & 1 \\
\hline Evergreen $^{\mathrm{B}}$ & -0.344 & -0.653 & 0 \\
\hline Snowdepth ${ }^{\mathrm{B}}$ & -0.001 & 0 & 0 \\
\hline Edge Density $^{\mathrm{B}}$ & 0 & 0 & 0 \\
\hline Cropland $^{\mathrm{B}}$ & 0.003 & 0 & 0.06 \\
\hline Grassland $^{\mathrm{B}}$ & 0.008 & 0 & 0.153 \\
\hline Deciduous $^{\mathrm{B}}$ & -0.005 & -0.091 & 0 \\
\hline Logit Intercept, r & -4.359 & -4.587 & -4.143 \\
\hline Trail & -0.166 & -0.504 & 0.172 \\
\hline Distance & 0.266 & 0.067 & 0.471 \\
\hline Distance $^{2}$ & -0.133 & -0.234 & -0.045 \\
\hline Spline Coefficient[1] & 0.01 & -0.503 & 0.753 \\
\hline Spline Coefficient[2] & -0.023 & -0.618 & 0.423 \\
\hline Spline Coefficient[3] & 0.008 & -0.267 & 0.648 \\
\hline Spline Coefficient[4] & -0.028 & -0.49 & 0.218 \\
\hline Spline Coefficient[5] & 0.001 & -0.542 & 0.506 \\
\hline Spline Coefficient[6] & 0.011 & -0.505 & 0.608 \\
\hline Spline Coefficient[7] & -0.01 & -0.559 & 0.519 \\
\hline Spline Coefficient[8] & 0.034 & -0.334 & 0.337 \\
\hline Spline Coefficient[9] & 0.016 & -0.478 & 0.602 \\
\hline Spline Coefficient[10] & 0.037 & -0.443 & 0.642 \\
\hline Spline Coefficient[11] & -0.111 & -0.85 & 0.162 \\
\hline Spline Coefficient[12] & 0.023 & -0.482 & 0.624 \\
\hline Spline Coefficient[13] & -0.004 & -0.488 & 0.4 \\
\hline Spline Coefficient[14] & 0.003 & -0.524 & 0.582 \\
\hline Spline Coefficient[15] & 0.052 & -0.353 & 0.702 \\
\hline Spline Coefficient[16] & 0.011 & -0.496 & 0.588 \\
\hline Spline Coefficient[17] & 0.025 & -0.444 & 0.649 \\
\hline Spline Coefficient[18] & 0.01 & -0.516 & 0.589 \\
\hline Spline Coefficient[19] & 0.03 & -0.443 & 0.694 \\
\hline Spline Coefficient[20] & 0.04 & -0.423 & 0.672 \\
\hline
\end{tabular}


Generalized false positive models

${ }^{A}$ Inclusion probability - marginal posterior probability that term was included within the model

${ }^{\mathrm{B}}$ Regularized coefficient. Unregularized coefficients include draws from the prior when the model term was not included within the model; regularized coefficients represent the iterative product of the raw coefficient and the indicator for inclusion. 
Generalized false positive models

Table S3. Parameter estimates, variable inclusion probabilities $(w)$, and $95 \%$ credible intervals (LCI, UCI) from a RN model for gray fox relative abundance accounting for false positive error.

\begin{tabular}{|c|c|c|c|}
\hline Parameter & Estimate & LCI & UCI \\
\hline Log intercept, $\lambda$ & -2.366 & -4.415 & 1.022 \\
\hline Evergreen & -0.641 & -4.228 & 4.132 \\
\hline Snowdepth & 0.033 & -6.062 & 6.118 \\
\hline Edge Density & 0.217 & -5.297 & 5.485 \\
\hline Cropland & -0.088 & -5.914 & 5.913 \\
\hline Grassland & -0.011 & -6.025 & 6.122 \\
\hline Deciduous & -0.272 & -5.292 & 5.111 \\
\hline Logit Intercept, $\mathrm{s}_{0}$ & -6.486 & -6.67 & -6.311 \\
\hline Cropland, $\mathrm{s}_{0}$ & 0.289 & 0.135 & 0.439 \\
\hline Evergreen, $\mathrm{w}^{\mathrm{A}}$ & 0.731 & 0 & 1 \\
\hline Snowdepth, $w^{A}$ & 0.102 & 0 & 1 \\
\hline Edge Density, w ${ }^{\mathrm{A}}$ & 0.451 & 0 & 1 \\
\hline Cropland, $\mathrm{w}^{\mathrm{A}}$ & 0.198 & 0 & 1 \\
\hline Grassland, $\mathrm{w}^{\mathrm{A}}$ & 0.088 & 0 & 1 \\
\hline Deciduous, $\mathrm{w}^{\mathrm{A}}$ & 0.493 & 0 & 1 \\
\hline Evergreen $^{\mathrm{B}}$ & -0.642 & -1.664 & 0 \\
\hline Snowdepth ${ }^{\mathrm{B}}$ & 0.021 & 0 & 0.362 \\
\hline Edge Density ${ }^{\mathrm{B}}$ & 0.207 & 0 & 0.779 \\
\hline Cropland $^{\mathrm{B}}$ & -0.068 & -0.59 & 0 \\
\hline Grassland $^{\mathrm{B}}$ & -0.017 & -0.313 & 0 \\
\hline Deciduous $^{B}$ & -0.244 & -0.861 & 0 \\
\hline Logit Intercept, r & -3.514 & -3.825 & -3.237 \\
\hline Trail & -0.384 & -0.983 & 0.195 \\
\hline Distance & 0.668 & 0.344 & 1.019 \\
\hline Distance $^{2}$ & -0.218 & -0.495 & 0.191 \\
\hline Spline Coefficient[1] & 0.008 & -0.565 & 0.519 \\
\hline Spline Coefficient[2] & -0.03 & -0.657 & 0.511 \\
\hline Spline Coefficient[3] & -0.114 & -0.632 & 0.184 \\
\hline Spline Coefficient[4] & 0.016 & -0.285 & 0.366 \\
\hline Spline Coefficient[5] & 0.003 & -0.607 & 0.595 \\
\hline Spline Coefficient[6] & 0.013 & -0.596 & 0.637 \\
\hline Spline Coefficient[7] & -0.007 & -0.618 & 0.598 \\
\hline Spline Coefficient[8] & 0.005 & -0.528 & 0.41 \\
\hline Spline Coefficient[9] & 0.013 & -0.591 & 0.635 \\
\hline Spline Coefficient[10] & 0.032 & -0.526 & 0.66 \\
\hline Spline Coefficient[11] & -0.079 & -0.718 & 0.352 \\
\hline Spline Coefficient[12] & 0.01 & -0.606 & 0.622 \\
\hline Spline Coefficient[13] & -0.009 & -0.538 & 0.534 \\
\hline Spline Coefficient[14] & -0.003 & -0.62 & 0.605 \\
\hline Spline Coefficient[15] & 0.004 & -0.61 & 0.587 \\
\hline Spline Coefficient[16] & 0.001 & -0.604 & 0.61 \\
\hline
\end{tabular}


Generalized false positive models

$\begin{array}{lrrr}\text { Spline Coefficient[17] } & -0.002 & -0.628 & 0.593 \\ \text { Spline Coefficient[18] } & 0.002 & -0.61 & 0.613 \\ \text { Spline Coefficient[19] } & -0.003 & -0.629 & 0.59 \\ \text { Spline Coefficient[20] } & 0.008 & -0.6 & 0.602\end{array}$

${ }^{\mathrm{A}}$ Inclusion probability—marginal posterior probability that term was included within the model

${ }^{\mathrm{B}}$ Regularized coefficient. Unregularized coefficients include draws from the prior when the model term was not included within the model; regularized coefficients represent the iterative product of the raw coefficient and the indicator for inclusion. 